\title{
The Nebulin Family LIM and SH3 Proteins Regulate Postsynaptic Development and Function
}

\author{
Kenneth R. Myers, ${ }^{1}$ Kuai Yu, ${ }^{1}$ Joachim Kremerskothen, ${ }^{4}$ Elke Butt, ${ }^{5}$ and $\odot$ James Q. Zheng ${ }^{1,2,3}$ \\ Departments of ${ }^{1}$ Cell Biology, ${ }^{2}$ Neurology, ${ }^{3}$ Center for Neurodegenerative Diseases, Emory University School of Medicine, Atlanta, Georgia 30322, \\ ${ }^{4}$ Medizinische Klinik D, Abteilung für Molekulare Nephrologie, Universitätsklinikum Münster, 48149 Münster, Germany, and ${ }^{5}$ Institute of Experimental \\ Biomedicine II, University Medical Clinic of Wuerzburg, 97080 Wuerzburg, Germany
}

Neuronal dendrites have specialized actin-rich structures called dendritic spines that receive and integrate most excitatory synaptic inputs. The stabilization of dendrites and spines during neuronal maturation is essential for proper neural circuit formation. Changes in dendritic morphology and stability are largely mediated by regulation of the actin cytoskeleton; however, the underlying mechanisms remain to be fully elucidated. Here, we present evidence that the nebulin family members LASP1 and LASP2 play an important role in the postsynaptic development of rat hippocampal neurons from both sexes. We find that both LASP1 and LASP2 are enriched in dendritic spines, and their knockdown impairs spine development and synapse formation. Furthermore, LASP2 exerts a distinct role in dendritic arbor and dendritic spine stabilization. Importantly, the actin-binding N-terminal LIM domain and nebulin repeats of LASP2 are required for spine stability and dendritic arbor complexity. These findings identify LASP1 and LASP2 as novel regulators of neuronal circuitry.

Key words: actin cytoskeleton; dendrite; neuronal development; spines; synapse

\section{Significance Statement}

Proper regulation of the actin cytoskeleton is essential for the structural stability of dendrites and dendritic spines. Consequently, the malformation of dendritic structures accompanies numerous neurologic disorders, such as schizophrenia and autism. Nebulin family members are best known for their role in regulating the stabilization and function of actin thin filaments in muscle. The two smallest family members, LASP1 and LASP2, are more structurally diverse and are expressed in a broader array of tissues. While both LASP1 and LASP2 are highly expressed in the brain, little is currently known about their function in the nervous system. In this study, we demonstrate the first evidence that LASP1 and LASP2 are involved in the formation and long-term maintenance of dendrites and dendritic spines.

\section{Introduction}

The structure of the dendritic arbor of a neuron is essential for neural circuit formation, determining how synaptic inputs are received and integrated. In turn, presynaptic inputs themselves can shape and stabilize the dendritic arbor (Parrish et al., 2007). The arbor is highly dynamic during early development as numerous branches form, elongate, and retract (Scott and Luo, 2001;

Received Feb. 11, 2019; revised Nov. 12, 2019; accepted Nov. 15, 2019.

Author contributions: K.R.M. and J.Q.Z. designed research; K.R.M. and K.Y. performed research; J.K. and E.B. contributed unpublished reagents/analytic tools; K.R.M. and J.Q.Z. analyzed data; K.R.M. wrote the paper

This work is supported in part by National Institutes of Health Grants MH-104632 and GM-083889 to J.Q.Z., and a Ruth L. Kirschstein National Research Service Awards Postdoctoral Fellowship (NS092342) to K.R.M. We thank Jinny Yoo and Jenny Mai for help with image analysis, and Madeline Morgan for helpful discussions.

The authors declare no competing financial interests.

Correspondence should be addressed to James Q. Zheng at james.zheng@emory.edu or Kenneth R. Myers at kennethmyers@emory.edu.

https://doi.org/10.1523/JNEUROSCI.0334-19.2019

Copyright $\odot 2020$ the authors
Parrish et al., 2007; Koleske, 2013). However, as development proceeds, increased neuronal activity stabilizes individual branches, and less active branches retract (Van Aelst and Cline, 2004; Cline and Haas, 2008). The majority of excitatory inputs are received at glutamatergic synapses, which are formed between presynaptic axonal terminals and specialized postsynaptic structures called dendritic spines (Nimchinsky et al., 2002; Bourne and Harris, 2008; Rochefort and Konnerth, 2012). Spines are small membranous protrusions that form on dendrites and function as the sites of signal integration and transduction downstream of glutamate receptor activity (Chen and Sabatini, 2012). Dendritic spines are highly plastic, and changes in their morphology underlie synapse formation and modification during learning and memory (Segal, 2005; Bosch and Hayashi, 2012; Lai and Ip, 2013). The growth of the dendritic arbor and dendritic spines are therefore closely linked, and both are essential for the development and function of neuronal circuits (Koleske, 2013). How- 
ever, as neurons mature, the dendritic arbor typically stabilizes while spines retain a reduced level of plasticity.

Changes in spine morphology are driven largely by remodeling of the actin cytoskeleton (Hotulainen and Hoogenraad, 2010; Lei et al., 2016). Actin filaments are remodeled by regulatory proteins that arrange actin into distinct networks with different functions. These regulatory proteins modify actin structures by crosslinking, severing, end capping, and bundling actin filaments. The LIM and SH3 domain-containing proteins LASP1 and LASP2 are small multidomain actin-binding proteins that have been implicated in the stabilization of actin filaments (Grunewald and Butt, 2008; Pappas et al., 2011). Consequently, LASP1 and LASP2 localize to numerous sites of dynamic actin assembly and disassembly, including focal adhesions, membrane ruffles, lamellipodia, and pseudopodia (Chew et al., 2002b). In non-neuronal cells, both LASP proteins have been shown to regulate cell adhesion and cell migration (Lin et al., 2004; Bliss et al., 2013). LASP1 and LASP 2 are highly homologous and are differentiated from the other members of the nebulin family of actinbinding proteins by their small size and the presence of an $\mathrm{N}$-terminal LIM domain (Pappas et al., 2011). LIM domains participate in a wide variety of cellular functions, including the regulation of gene expression, signal transduction, cell adhesion, and cell motility (Kadrmas and Beckerle, 2004). In addition, LASP1 and LASP2 feature a C-terminal Src homology region 3 (SH3) domain, a flexible nonstructured linker region, and either two or three 35 aa actin-binding nebulin-like repeats, respectively (Grunewald and Butt, 2008). This unique domain architecture enables the LASP proteins to serve as signaling hubs, linking numerous proteins to the actin cytoskeleton (Orth et al., 2015). Studies have shown that both proteins are highly expressed in the CNS, and that LASP1 is concentrated in the postsynaptic density (Phillips et al., 2004; Terasaki et al., 2004). In addition, LASP1 has been identified as a risk factor for both autism and schizophrenia (Stone et al., 2007; Joo et al., 2013). However, little is currently known about the physiological role of either LASP1 or LASP2 in the nervous system.

In this study, we present evidence that LASP1 and LASP2 play an important role in dendritic spine development and stabilization, respectively. Furthermore, we demonstrate that LASP2, but not LASP1, promotes dendritic complexity by stabilizing dendritic branches. LASP2, but not LASP1, has been identified as an actin-bundling protein (Chew et al., 2002a; Zieseniss et al., 2008), and here we identify a role for the actin-binding LIM domain and nebulin repeats in LASP2-mediated dendritic stabilization. Our findings demonstrate an important new role for nebulin family members in the stabilization of dendritic spines and dendritic arbors, and in regulating synapse formation.

\section{Materials and Methods}

Antibodies. The following commercial antibodies were used: rabbit antimicrotubule-associated protein 2 (MAP2; 1:1000; catalog \#AB5622, EMD Millipore), rabbit anti-red fluorescent protein (RFP; 1:1000; cata$\log \# 600-401-279$, Rockland), rabbit anti-green fluorescent protein (GFP; 1:1000; catalog \#A-11122; Thermo Fisher Scientific), mouse antipostsynaptic density protein 95 (PSD-95; 1:1000; MA1-045; Thermo Fisher Scientific), and mouse anti-SV2 (synaptic vesicle 2; 1:1000; Developmental Studies Hybridoma Bank, Iowa City, IA). To examine LASP localization, we used a total LASP antibody (i.e., recognizes both LASP1 and LASP2), which was generated by immunizing rabbit with full-length GST-LASP1 (Butt et al., 2003). For Western blotting of LASP1 specifically, we used mouse monoclonal anti-LASP1 clone-B8, which was generated against the following sequence within the LASP1 linker domain (not present in LASP2): SYRRPLEQQQPH (amino acids 151-162; Butt and Raman, 2018).

DNA constructs. GFP-LASP1 constructs [wild-type (WT), $\Delta$ LIM, $\Delta$ Linker, and $\Delta \mathrm{SH} 3]$ were described previously (Stölting et al., 2012). GFP-LASP1- $\Delta$ Nebulin was prepared by removing amino acids $62-128$ from wild-type GFP-LASP1 constructs. GFP-LASP2 constructs were generated by subcloning de novo synthesized human LASP2 into the BglII/EcoRI sites of pEGFP-C1, as follows: $\Delta$ LIM lacks amino acids 4-56, $\Delta$ Nebulin lacks amino acids $62-128, \Delta$ Linker lacks amino acids $168-209$, and $\Delta$ SH3 lacks amino acids 213-270. mCherry-pSuper was created by removing EGFP from pSuper.neo + GFP using the NheI/BspTI sites, and subcloning mCherry from pmCherry-C1. To create two shRNAs each against LASP1 (shLASP1a and shLASP1b) and LASP2 (shLASP2a and shLASP2b), the following oligos were synthesized, ligated, and then inserted into pSuper.neo + mCherry using the BglII/XhoI sites: shLASP1a, 5'GATCCCCCCATTAAGGAGATCGGTTATTCAAGAGATAACCGA TCTCCTTAATGGTTTTTC (Forward)/ 5'TCGAGAAAAACCATTAA GGAGATCGGTTATCTCTTGAATAACCGATCTCCTTAATGGGGG (Reverse); shLASP1b, 5'GATCCCCGACCAATCCTGTAGCGCAATTC AAGAGATTGCGCTACAGGATTGGTCTTTTTC (Forward)/ 5'TCG AGAAAAAGACCAATCCTGTAGCGCAATCTCTTGAATTGCGCTAC AGGATTGGTCGGG (Reverse); shLASP2a, 5'GATCCCCCAGCGATG CTGCCTATAAATTCAAGAGATTTATAGGCAGCATCGCTGTTTT TC (Forward)/ 5'TCGAGAAAAACAGCGATGCTGCCTATAAAT CTCTTGAATTTATAGGCAGCATCGCTGGGG (Reverse); and shLASP2b, 5'GATCCCCCAATGCAGCATTCACCAAATTCAAGAG ATTTGGTGAATGCTGCATTGTTTTTC (Forward)/5'TCGAGAAAA ACAATGCAGCATTCACCAAATCTCTTGAATTTGGTGAATGCTGC ATTGGGG (Reverse). mOrange-actin was created by subcloning mOrange into phosphorylated EGFP (pEGFP)-actin (Clontech), and the PSD-95-GFP and PSD-95-dsRed constructs were provided by Dr. Bonnie Firestein (Rutgers University, New Brunswick, NJ).

Neuronal culture and transfection. Timed-pregnant Sprague Dawley rats were obtained from Charles River Laboratories. Dissociated primary hippocampal neuron cultures were prepared from embryonic day 18.5 rat embryos of both sexes. Brains were removed and hippocampi were dissected in cold HBSS. Hippocampi were trypsinized, triturated, and then plated on $25 \mathrm{~mm}$ coverslips coated with 100 $\mu \mathrm{g} / \mathrm{ml}$ poly-L-lysine (Sigma-Aldrich) at a density of $\sim 325,000$ cells per $35 \mathrm{~mm}$ dish. Neurons were maintained in Neurobasal Medium supplemented with B-27 (Thermo Fisher Scientific), penicillin/streptomycin, and Invitrogen GlutaMax (Thermo Fisher Scientific). Neurons were transfected using Calphos calcium phosphate transfection reagent (Takara) on the specified days. Animal care and use were performed in accordance with the guidelines of the National Institutes of Health, and were approved by the Institutional Animal Care and Use Committee at Emory University.

Immunocytochemistry. For immunostaining, hippocampal neurons were fixed on the specified date with freshly prepared $4 \%$ paraformaldehyde plus 4\% sucrose in PBS for $15 \mathrm{~min}$ at room temperature. Neurons were washed in PBS and then blocked and permeabilized in PBS containing $1 \%$ BSA, $5 \%$ normal goat serum, and $0.1 \%$ Triton $\mathrm{X}-100$ for $1 \mathrm{~h}$ at room temperature. Then neurons were incubated with primary antibodies for $1 \mathrm{~h}$, washed, incubated with fluorescent Alexa Fluor (Alexa Fluor488, Alexa Fluor-546, or Alexa Fluor-647; 1:750; Thermo Fisher Scientific) secondary antibodies for $1 \mathrm{~h}$, washed, and mounted on slides with Fluoromount-G (Southern Biotech). All antibodies were diluted into blocking/permeabilization buffer. Imaging was performed using a Nikon C1 laser-scanning confocal system with an inverted Nikon TE300 (60× Apo TIRF (total internal reflection fluorescence) objective, 1.49 numerical aperture (NA)], a Nikon C2 laser-scanning confocal system with an inverted Nikon Ti2 microscope (60× Plan Apo objective, 1.4 NA), or an epifluorescent Nikon Eclipse Ti inverted (20× Plan Fluor objective, $0.5 \mathrm{NA}$ ).

Live-cell imaging. For live-cell imaging, neurons were cultured in fourwell glass-bottomed dishes coated with $100 \mu \mathrm{g} / \mathrm{ml}$ poly-L-lysine at a density of $\sim 50,000$ cells/well. Cells were cultured in phenol-red free Neurobasal medium supplemented as described above. For imaging, dishes were placed on the microscope stage and housed at $37^{\circ} \mathrm{C}$ in $5 \%$ 
a
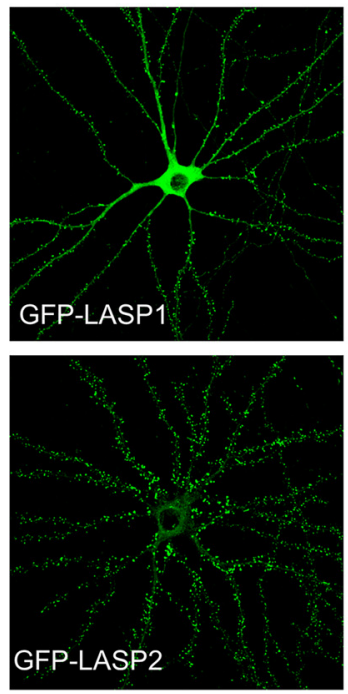
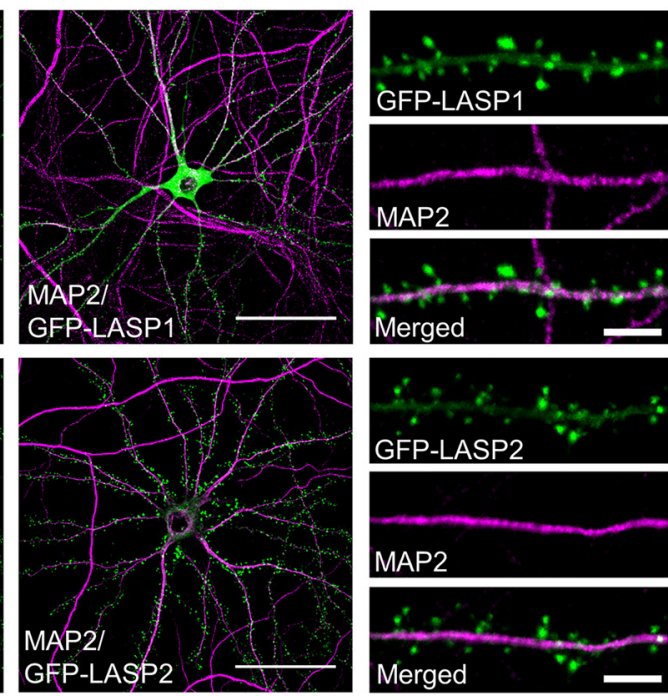

b

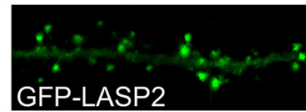

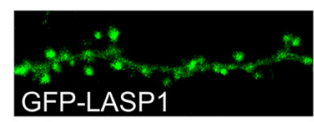
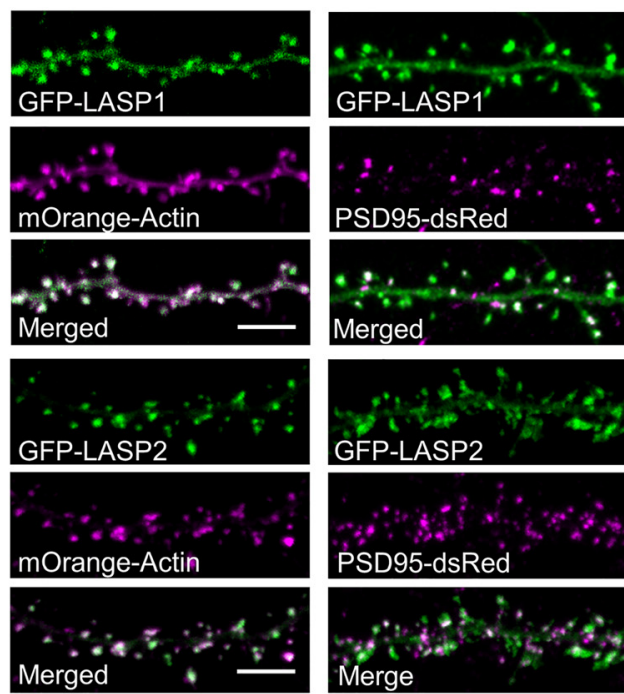

C

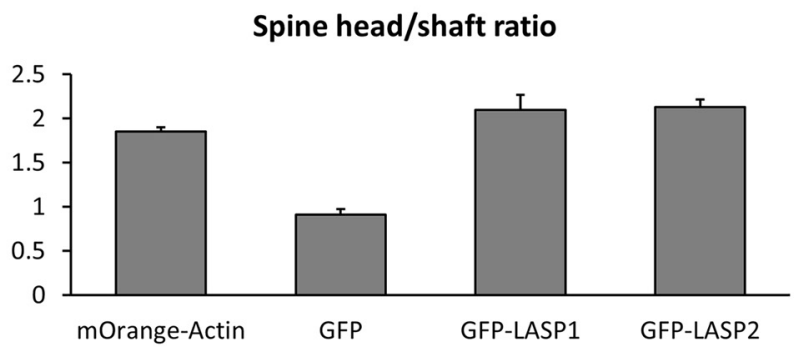

d

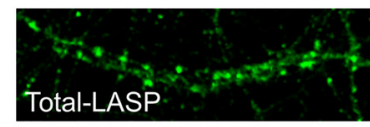

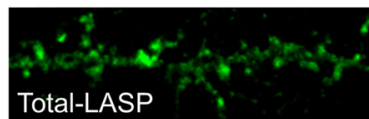
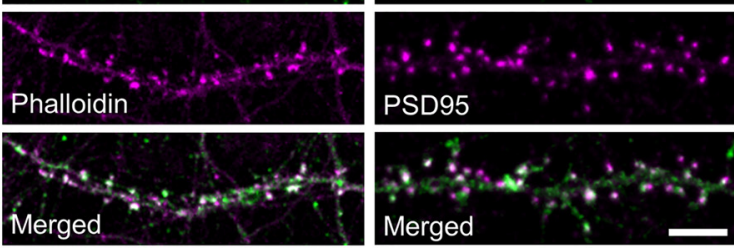

Figure 1. LASP proteins are specifically enriched in dendritic spines. $\boldsymbol{a}$, Representative images of rat hippocampal neurons at DIV21 showing localization of GFP-tagged LASP1 and LASP2 (green) in dendrites highlighted by MAP2 (magenta). Scale bars: left, $50 \mu \mathrm{m}$; inset, right, $5 \mu \mathrm{m}$. $\boldsymbol{b}$, Representative images showing enrichment of GFP-LASP1 or GFP-LASP2 (green) in dendritic spines and the postsynaptic density, highlighted by m0range-actin or the excitatory synaptic marker PSD-95-dsRed, respectively (magenta). Scale bars, $5 \mu$ m. c, Enrichment of LASPs in spines as measured by the ratio of fluorescent intensity detected in the spine head versus the intensity in the neighboring dendritic shaft. Error bars represent the SEM ( $n=156$ spines from 13 cells for mOrange-actin, 156 spines from 13 cells for GFP, 120 spines from 10 cells for GFP-LASP1, and 156 spines from 13 cells for GFP-LASP2. Data were collected from three independent experiments. $\boldsymbol{d}$, Endogenous total-LASP distribution (green) in spines costained for endogenous F-actin (568-phalloidin, magenta) or endogenous PSD-95 (magenta). Scale bars, $5 \mu \mathrm{m}$.

$\mathrm{CO}_{2}$ in a humidified temperature- and $\mathrm{CO}_{2}$-controlled chamber (TokaiHit). Cells were imaged using a Nikon Eclipse Ti inverted microscope with a Perfect Focus System and a $20 \times$ objective (Plan Fluor, $0.5 \mathrm{NA}$ ) for imaging dendritic branches, and a $60 \times$ objective (Apo TIRF, $1.49 \mathrm{NA}$ ) with $1.5 \times$ zoom for imaging spines.

Image analysis. All samples were blinded before image acquisition and revealed after image analysis was completed. Imaging of spines was conducted using a Nikon laser-scanning confocal microscope with a $60 \times$ objective and $3 \times$ digital zoom. $Z$-stacks composed of 14-24 optical sections $(0.2 \mu \mathrm{m}$ steps) were acquired, and maximum intensity projections of $Z$-stacks were used to generate $2 \mathrm{D}$ images. ImageJ and Imaris software were used for visualizing and quantifying spine density and morphology measurements. Dendritic spine number, length, and width for each neuron were averaged from two segments at least $30 \mu \mathrm{m}$ in length from different secondary or tertiary branches. Filopodial-like spines were defined as having a length $\geq 2 \mu \mathrm{m}$, with no discernable spine head. Nikon Elements software was used to perform semiautomated analysis of the spine head/shaft ratio after manually identifying dendritic spines. Synapse density was calculated manually by counting all PSD-95 puncta that directly coclustered with SV2 puncta and had an average fluorescence intensity at least twice as high as the neighboring dendritic shaft. Imaging dendritic arbors was typically conducted using an epifluorescent Nikon Eclipse Ti inverted microscope with a $20 \times$ objective (Plan Fluor, 0.5 NA), and all tracing and Sholl analysis were performed using the Simple Neurite Tracer ImageJ plugin (Longair et al., 2011). Analysis of dendrite branches from live cells was performed using the Manual Tracking ImageJ plugin. All data represent at least three replicates from independently prepared samples. The number of cells, dendritic spines, or dendritic branches analyzed are reported in the corresponding figure legend. GraphPad Prism version 7 (GraphPad Software) was used for statistical analysis. Data are presented as the mean \pm SEM (standard error of the mean) or the mean $\pm 95 \%$ CI (confidence interval).

Electrophysiology. Whole-cell patch-clamp recordings were performed using an EPC7 amplifier (HEKA). Patch pipettes were pulled from borosilicate glass and fire polished (4-6 M $\Omega$ ). The recording chamber was continuously perfused with HEPES-buffered recording solution (128 $\mathrm{mm} \mathrm{NaCl}, 5 \mathrm{~mm} \mathrm{KCl}, 2 \mathrm{~mm} \mathrm{CaCl} 2,1 \mathrm{~mm} \mathrm{MgCl}_{2}, 25 \mathrm{~mm}$ HEPES, and 30 $\mathrm{mm}$ glucose, $\mathrm{pH} 7.3$ adjusted with $\mathrm{NaOH}$, and osmolarity at $310-320$

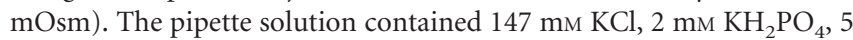
mм Tris-HCl, 2 mм EGTA, 10 mм HEPES, 4 mm Mg-ATP, and $0.5 \mathrm{~mm}$ $\mathrm{Na}_{2} \mathrm{GTP}, \mathrm{pH} 7.3$ adjusted with $\mathrm{KOH}$, and osmolarity at 310-320 mOsm. The membrane potential was clamped at $-70 \mathrm{mV}$. Data were acquired using pClamp 9 software, sampled at $5 \mathrm{kHz}$, and filtered at $1 \mathrm{kHz}$. For miniature EPSC (mEPSC) recordings, $0.5 \mu \mathrm{M}$ TTX and $100 \mu \mathrm{M}$ picrotoxin were added to block action potentials and $\mathrm{GABA}_{\mathrm{A}}$ receptors. Offline data analysis was performed using Clampfit 9.0 software (Molecular Devices), and events were detected automatically using a $5 \mathrm{pA}$ baseline.

Experimental design and statistical analysis. All data represent at least three replicates from independently prepared samples, and the total number of spines and/or cells used per condition is provided in the figure legends. Most data were analyzed using a Kruskal-Wallis one-way ANOVA with a Dunn's multiple-comparison test for nonparametric data or a two-way ANOVA with Tukey's post hoc test. GraphPad Prism (version 7, GraphPad Software) was used for statistical analysis, and results are provided in the figure legends. Unless otherwise specified, data are presented as the mean \pm SEM, with in-text values stated. The $p$ values are presented as ${ }^{\star} p \leq 0.05,{ }^{* *} p \leq 0.01,{ }^{* *} p \leq 0.001$. 


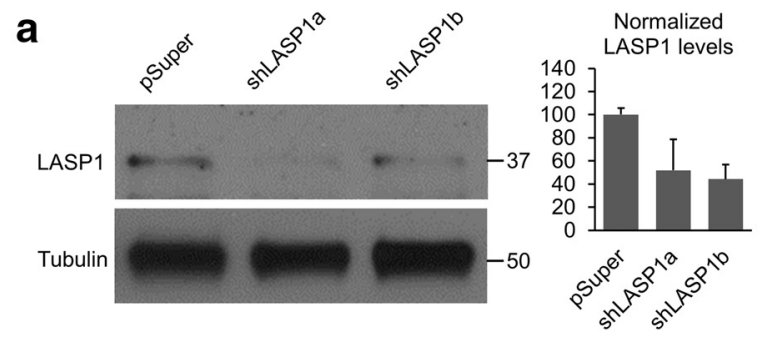

C

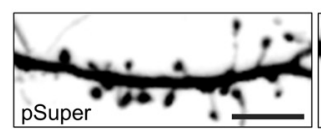

Spines / $10 \mu \mathrm{m}$
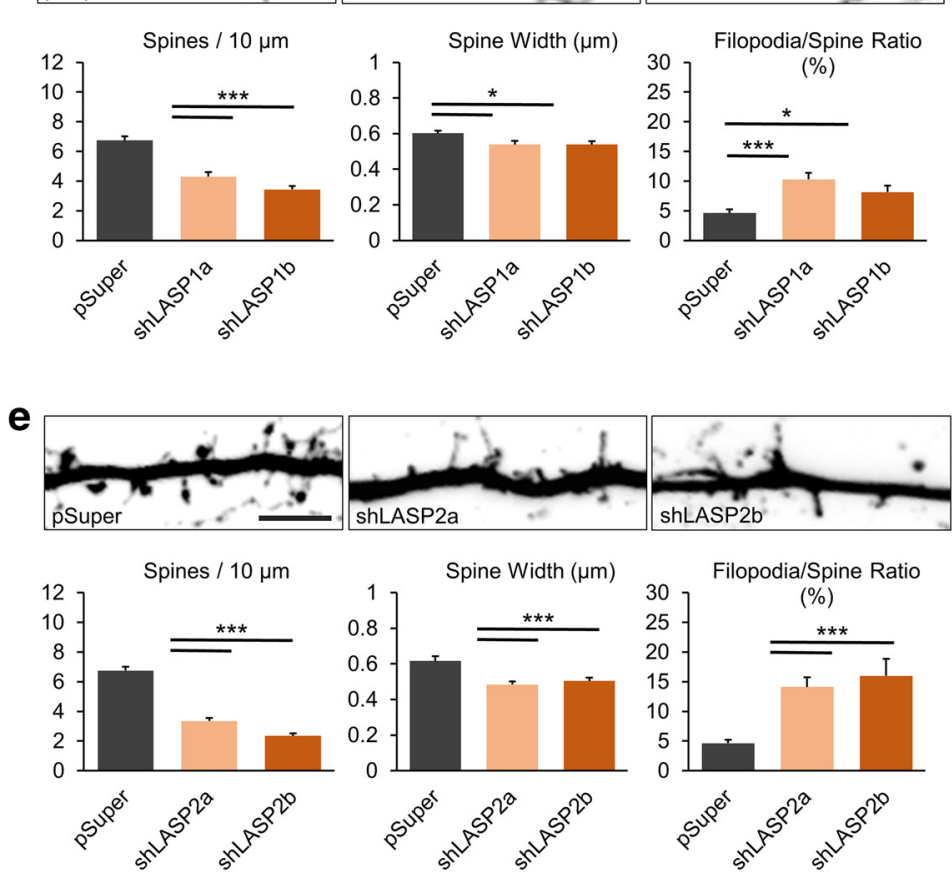

b
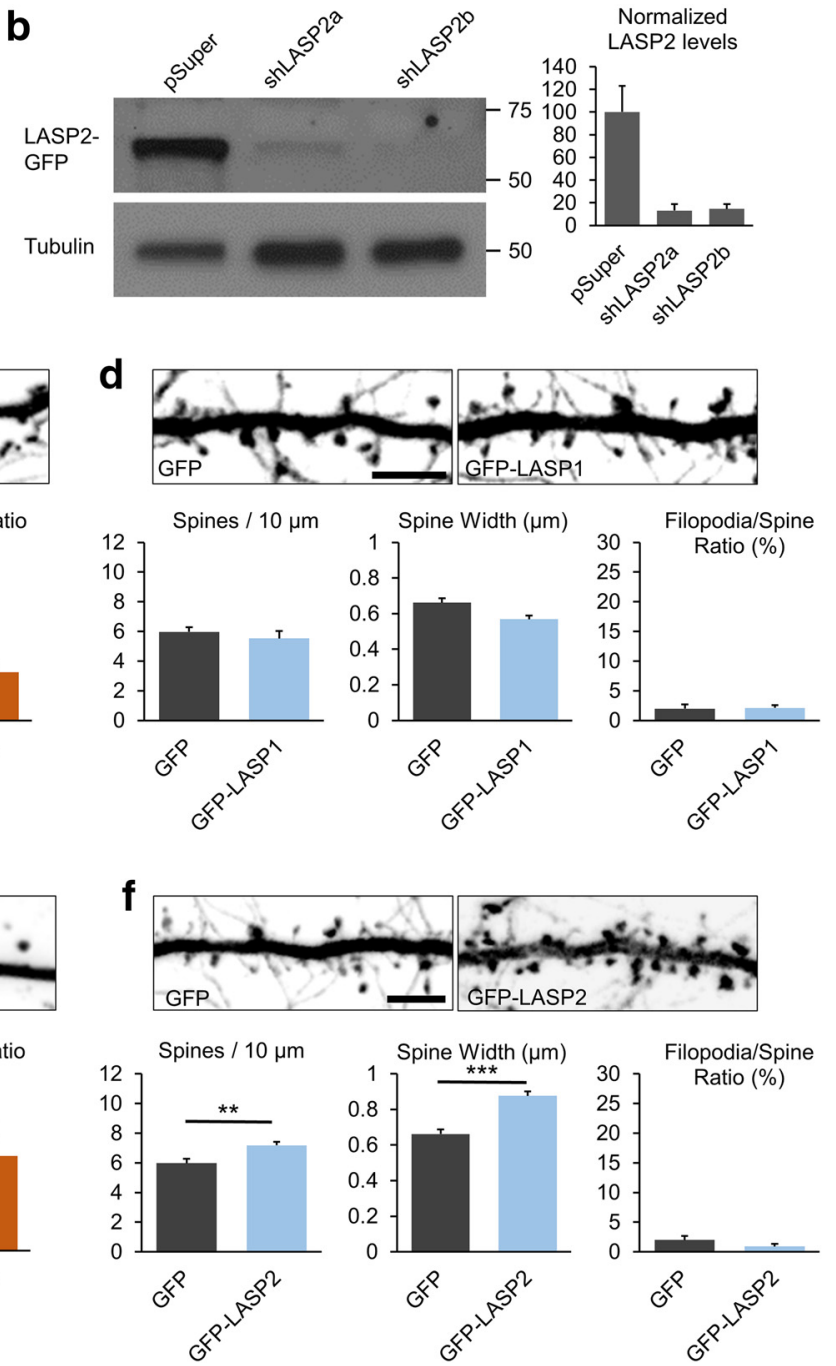

Figure 2. LASP1 and LASP2 regulate spine development and synapse formation. $\boldsymbol{a}, \boldsymbol{b}$, Left, Representative Western blots from cortical neurons expressing LASP1 and LASP2 shRNA constructs for $72 \mathrm{~h}$ showing either endogenous LASP1 ( $\boldsymbol{a}$ ) or GFP-LASP2 ( $\boldsymbol{b}$ ) protein levels along with tubulin as a loading control. Right, Bar graphs show mean LASP1 and LASP2 levels from three independent experiments. Data are normalized to pSuper control cells. $c$, Top, Representative images of dendritic spines from DIV21 hippocampal neurons transfected on DIV10 with pSuper, shLASP1a, and shLASP1b. Scale bar, $5 \mu \mathrm{m}$. Bottom, Quantification of spine density (Kruskal-Wallis test: $p<0.0001, \mathrm{H}=34.42, \mathrm{df}=2$; Dunn's test: $\mathrm{pSuper}$ vs shLASP1a, $p<0.0001 ;$;Super vs shLASP1b, $p<$ 0.0001 ), spine width (Kruskal-Wallis test: $p=0.0248, \mathrm{H}=7.395, \mathrm{df}=2$; Dunn's test: PSuper vs shLASP1a, $p=0.0492 ; \mathrm{pSuper}$ vs shLASP1b, $p=0.0286$ ), and the percentage of spines with filopodial-like morphology (Kruskal-Wallis test: $p<0.0001, \mathrm{H}=17.87, \mathrm{df}=2$; Dunn's test: $\mathrm{pSuper}$ vs shLASP1a, $p<0.0001 ; \mathrm{pSuper}$ vs shLASP1b, $p=0.0209 ; n=519$ spines from 23 cells for pSuper, 342 spines from 23 cells for shLASP1a, and 478 spines from 17 cells for shLASP1b). $\boldsymbol{d}$, Top, Representative images of dendritic spines from DIV21 hippocampal neurons transfected on DIV11 with GFP or GFP-LASP1. Scale bar, $5 \mu \mathrm{m}$. Bottom, Quantification of spine density (Mann-Whitney test: $p=0.4949, \mathrm{U}=224$ ), spine width (Mann-Whitney test: $p=0.55, \mathrm{U}=168$ ), and percentage of spines with filopodial-like morphology (Mann-Whitney test: $p=0.4813, \mathrm{U}=223 ; n=621$ spines from 23 cells for GFP, and 606 spines from 25 cells for GFP-LASP1). , Top, Images from DIV21 neurons transfected with pSuper, shLASP2a, and shLASP2b on DIV10. Scale bar, $5 \mu \mathrm{m}$. Bottom, Quantification of spine density (Kruskal-Wallis test: $p<0.0001, \mathrm{H}=25.9, \mathrm{df}=2$; Dunn's test: $\mathrm{pS}$ uper vs shLASP2a, $p<0.0001$; $\mathrm{pSuper}$ vs shLASP2b, $p<0.0001$ ), spine width (Kruskal-Wallis test: $p=0.0006, \mathrm{H}=14.74, \mathrm{df}=2 ; \mathrm{Dunn}$ 's test: $\mathrm{pS}$ Uper vs shLASP2a, $p=0.0004$; pSuper vs shLASP2b, $p=0.0171$ ), and percentage of spines with filopodial-like morphology (Kruskal-Wallis test: $p<0.0001, \mathrm{H}=25.9, \mathrm{df}=2 ; \mathrm{Dunn}$ 's test: $\mathrm{pSuper}$ vs shLASP2a, $p<0.0001$; pSuper vs shLASP2b, $p<0.0001 ; n=519$ spines from 23 cells for pSuper, 258 spines from 22 cells for shLASP2a, and 325 spines from 18 cells for shLASP1b). $\boldsymbol{f}$, Top, Representative images of dendritic spines from DIV21 hippocampal neurons transfected on DIV11 with GFP or GFP-LASP2. Scale bar, $5 \mu \mathrm{m}$. Bottom, Quantification of spine density (Mann-Whitney test: $p=0.0016, \mathrm{U}=$ 125), spine width (Mann-Whitney test: $p<0.0001, \mathrm{U}=53$ ), and percentage of spines with filopodial-like morphology (Mann-Whitney test: $p=0.4348, \mathrm{U}=234 ; n=1168$ spines from 18 cells for GFP, and 1179 spines from 17 cells for GFP-LASP2). All graphs represent data collected from three independent experiments. All error bars represent the SEM. ${ }^{*} p \leq 0.05,{ }^{* *} p \leq 0.01,{ }^{* * *} p \leq$ 0.001 by Kruskal-Wallis test with Dunn's post hoc test or Mann-Whitney test.

\section{Results}

\section{LASP1 and LASP2 are enriched in dendritic spines}

To understand the function of LASP1 and LASP2 in the nervous system, we first examined their subcellular localization in dissociated rat hippocampal neurons in culture. We expressed EGFP-tagged LASP1 and LASP2 in neurons at low levels and found that both proteins are generally present in the somatodendritic compartment, as marked by MAP2 (Fig. 1a). Strikingly, both LASP1 and LASP2 are enriched in dendritic spines, colocalizing with either fluorescently tagged actin or PSD-95, a key component of the postsynaptic density structure (Fig. $1 b$ ). This finding substantiates a previous proteomics study identifying LASP1 as a component of the postsynaptic density (Phillips et al., 2004) and suggests that both LASP1 and LASP2 are present in the postsynaptic compartment. It should be noted that we have observed a small percentage of cells where the levels of cytosolic GFP-LASP2 within the soma and dendritic shaft are extremely low compared with the levels in 
a

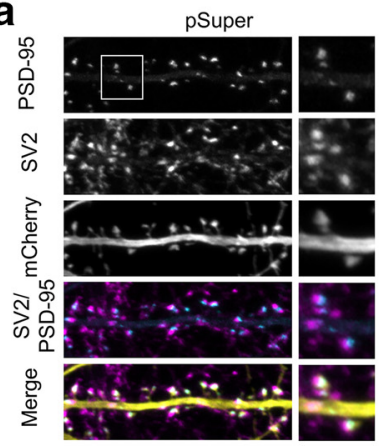

b
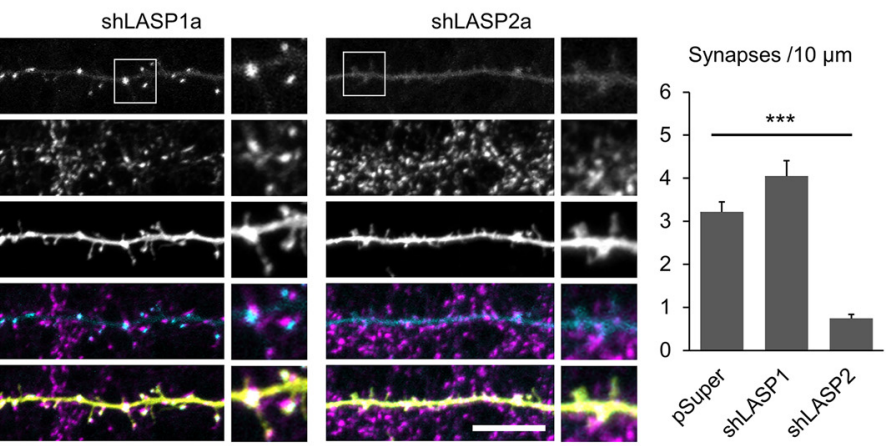

Control

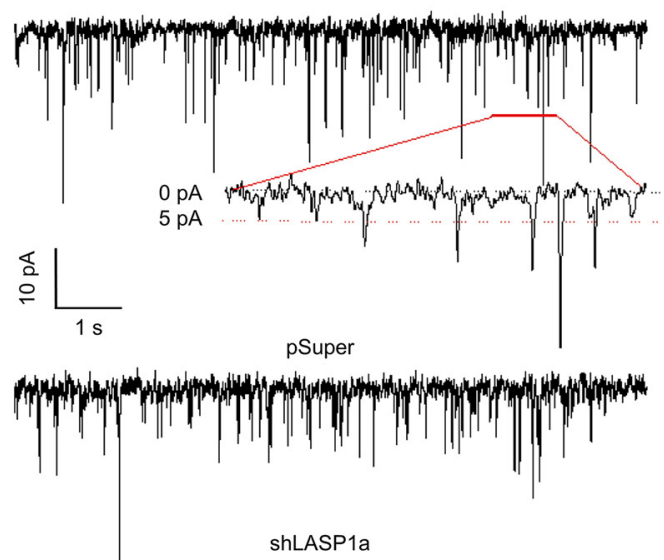

shLASP1a

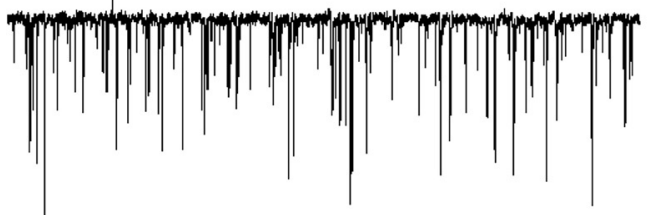

ShLASP2a

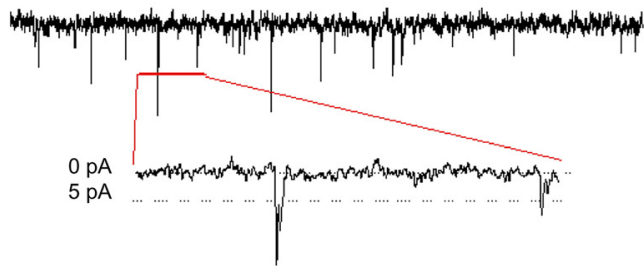

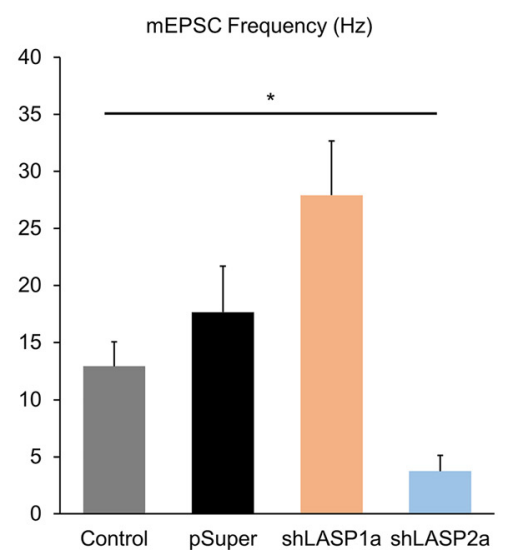

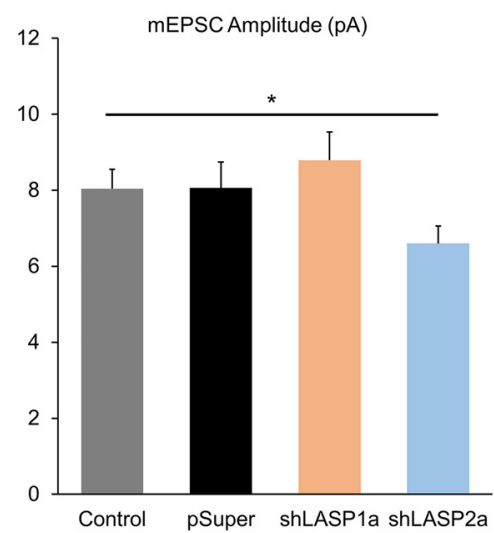

Figure 3. Regulation of synapse development and function by LASP2. $\boldsymbol{a}$, Left, Representative images of DIV 21 neurons cotransfected with the postsynaptic marker GFP-PSD-95 (magenta), and mCherry-pSuper, mCherry-shLASP1a, or mCherry-shLASP2a (yellow). Neurons were fixed and stained for endogenous SV2 (cyan), a presynaptic marker. Graph depicts the number of synapses (GFP-PSD-95/SV2 coclusters) per $10 \mu \mathrm{m}$ (Kruskal-Wallis test: $p<0.0001, \mathrm{H}=32.02$, df $=2$; Dunn's test: pSuper vs shLASP1a, $p=0.5718 ;$ pSuper vs shLASP2a, $p<0.0001 ; n=17$ cells pSuper, $n=18 \mathrm{shLASP1a}$, and $n=15 \mathrm{shLASP2a}$, collected from three independent experiments.). Scale bar, $10 \mu \mathrm{m}$. $\boldsymbol{b}$, Representative sample traces of mEPSCs from whole-cell patch-clamp recordings of DIV21 hippocampal neurons expressing pSuper, shLASP1a, shLASP2a, or neighboring nontransfected control cells. Histograms show mean mEPSC frequency (Kruskal-Wallis test: $p=0.0001$, $H=20.31$, df $=3$; Dunn's test: control vs $p S u p e r, p>0.9999$; control vs shLASP1a, $p=0.3990$; control vs shLASP2a, $p=0.0249$ ) and amplitude (Kruskal-Wallis test: $p=0.0078, H=11.89$, $\mathrm{df}=3$; Dunn's test: control vs pSuper, $p>0.9999$; control vs shLASP1a, $p>0.9999 ;$ control vs shLASP2a, $p=0.0274 ; n=8$ cells (ontrol, $n=5 \mathrm{pSuper}, n=7$ shLASP1a, and $n=13$ shLASP2a). Error bars represent the SEM. ${ }^{*} p \leq 0.05,{ }^{* * *} p \leq 0.001$ by Kruskal-Wallis test with Dunn's post hoc test.

spines. To quantify the enrichment of each protein in spines, we measured the ratio of GFP fluorescence in individual spine heads compared with the neighboring dendritic shaft $(\mathrm{H} / \mathrm{S}$ ratio) using confocal imaging. GFP-LASP1 and GFP-LASP2 have $\mathrm{H} / \mathrm{S}$ ratios of 2.5 and 2.6 , respectively, while soluble GFP has an $\mathrm{H} / \mathrm{S}$ ratio of $<1$ (Fig. 1c). This indicates that both GFP-LASP1 and GFP-LASP2 are preferentially enriched in dendritic spines. The enrichment of GFP-LASP1 and GFPLASP2 is not likely an artifact of exogenous overexpression, as we found that endogenous LASP proteins exhibit a similar spine enrichment when examined by immunocytochemistry (Fig. 1d). Using a total-LASP antibody that recognizes both LASP1 and LASP2, we found that endogenous LASP is enriched in spine heads and colocalizes with endogenous PSD-95 and F-actin. The lack of a LASP1- or LASP2-specific antibody suitable for immunocytochemistry prevented us from examining their endogenous distribution in neurons, individually. However, the total-LASP staining supports the use of low levels of exogenously expressed GFP-tagged LASP proteins to accurately represent the subcellular localization of LASPs. 

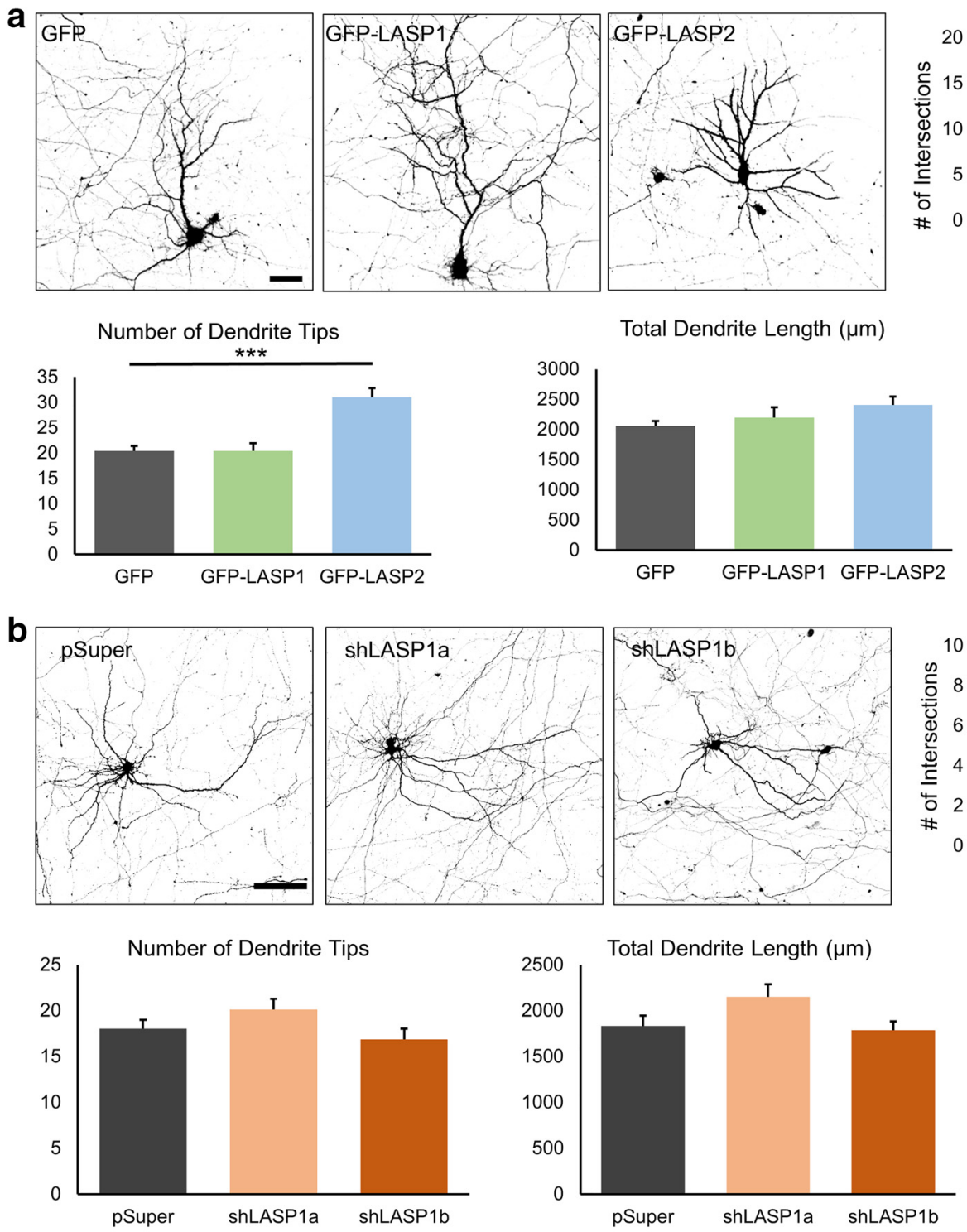

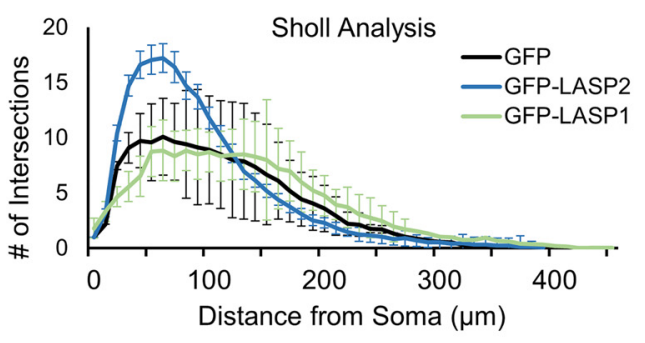

Total Dendrite Length $(\mu \mathrm{m})$
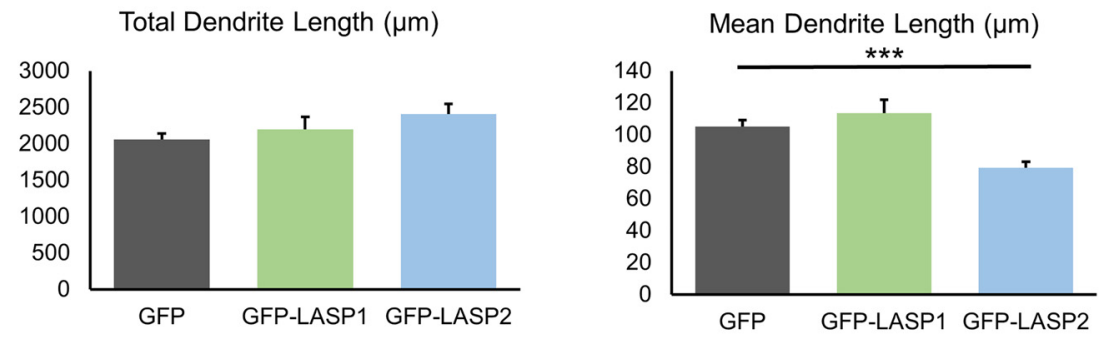

Sholl Analysis
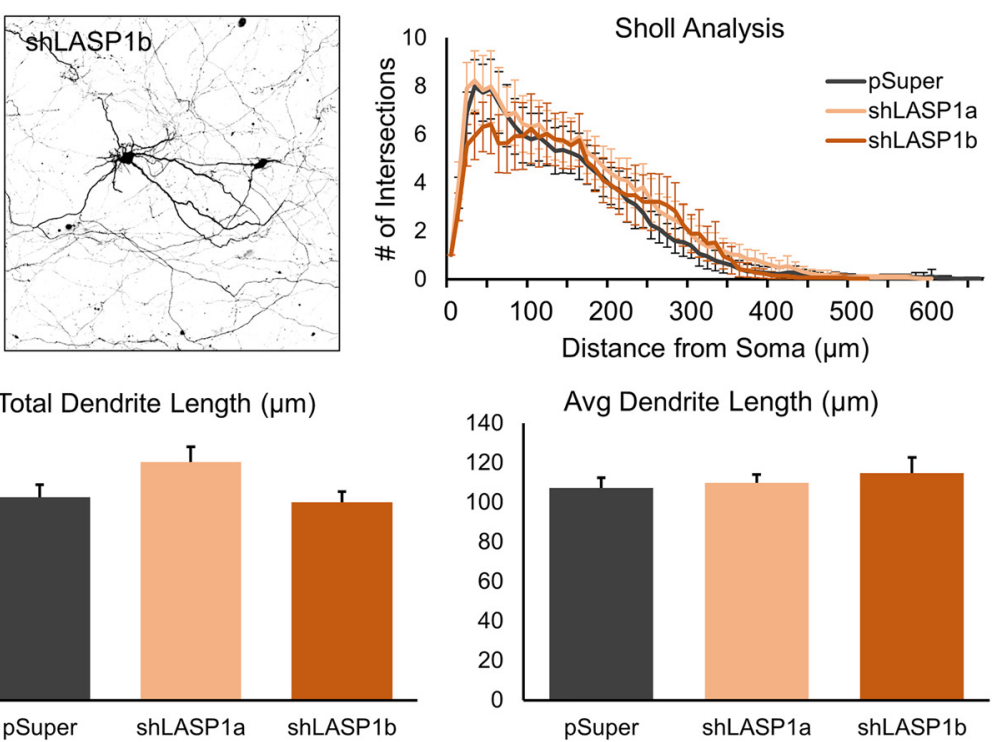
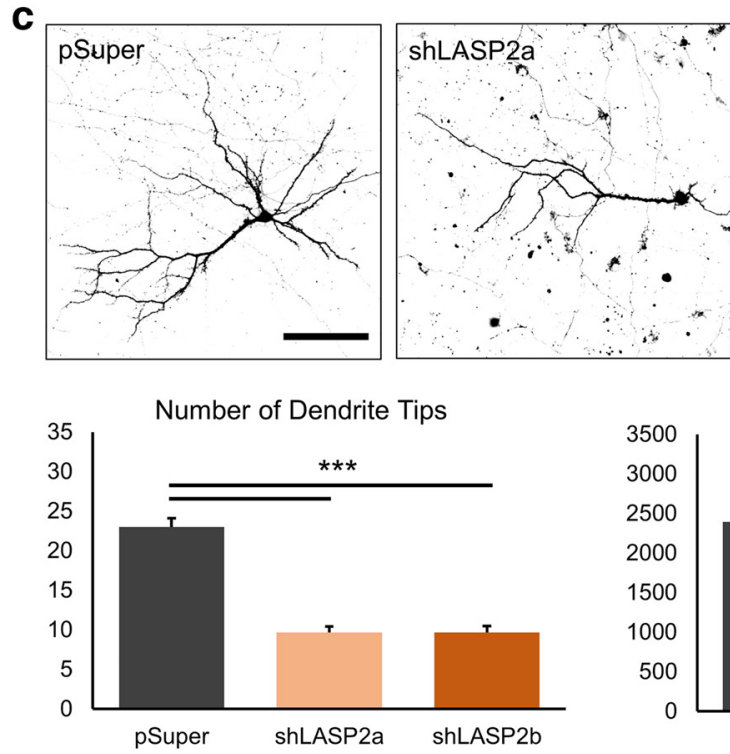

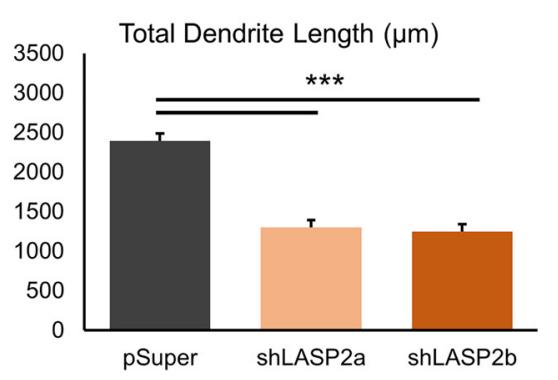

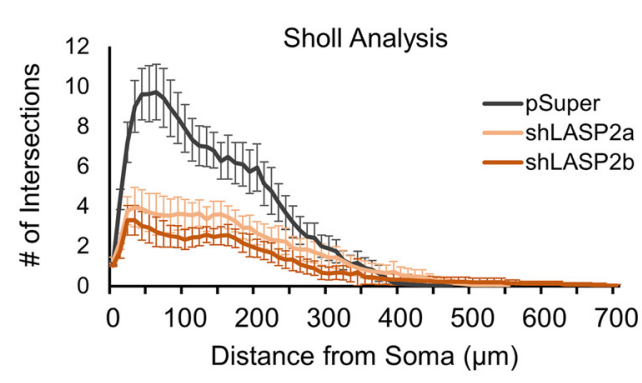

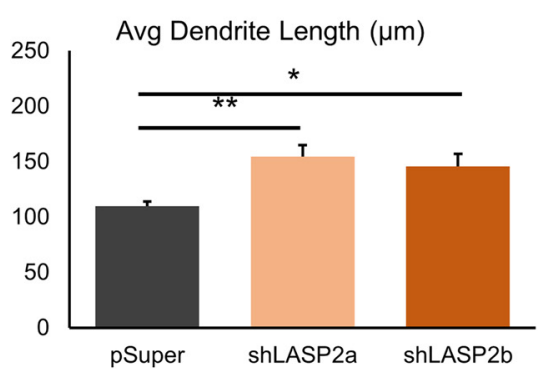

Figure 4. LASP2, but not LASP1, is required for normal dendritic arbor development. $\boldsymbol{a}$, Top left, Representative images of DIV21 hippocampal neurons expressing GFP, GFP-LASP1, or GFP-LASP2 for $9 \mathrm{~d}$ ( $n=35,31$, and 29, respectively). Top right, Sholl analysis shows the number of dendritic intersections for each condition plotted versus the distance from the center of the soma. Bottom, Graphs of the mean number of dendritic tips (Kruskal-Wallis test: $p<0.0001, \mathrm{H}=24.46, \mathrm{df}=2$; Dunn's test: GFP vs GFP-LASP1, $p>0.9999 ;$ GFP vs GFP-LASP2, (Figure legend continues.) 


\section{Knockdown of LASP1 and LASP2 diminishes spine development and synapse formation}

The enrichment of both LASP proteins in spines, together with their known functions in actin remodeling, suggested a role in dendritic spine development (Grunewald and Butt, 2008). Therefore, we examined the effects of LASP1 and LASP2 loss of function on spine development and morphology using shRNAmediated knockdowns. To control for off-target effects, we engineered two separate shRNAs each against LASP1 (plasmids shLASP1a and shLASP1b) and LASP2 (plasmids shLASP2a and shLASP2b). shLASP1a and shLASP1b target distinct nonoverlapping sequences within the 3' UTR of LASP1, while shLASP2a and shLASP2b target nonoverlapping sequences within the coding region of LASP2. shRNAs were expressed from an $\mathrm{H} 1$ promoter, and mCherry was expressed from a PGK (phosphoglycerate kinase) promoter using the pSuper.neo + mCherry (pSuper) vector. We evaluated knock-down efficiency in dissociated rat cortical neurons $72 \mathrm{~h}$ post-transfection by immunoblotting. Using a monoclonal antibody specific for LASP1, we found that both shLASP1a and shLASP1b markedly reduced endogenous LASP1 levels to $51.8 \pm 26.9 \%$ and $44.2 \pm 12.6 \%$ (mean \pm SEM) of pSuper control cells, respectively (Fig. $2 a$ ). Due to the lack of a LASP2-specific antibody, we were forced to evaluate LASP2 knock-down efficiency by examining the levels of cotransfected GFP-LASP2. We found that both shLASP2a and shLASP2b can efficiently knock down GFP-LASP2 to $13.1 \pm 5.6 \%$ and $14.6 \pm$ $4.2 \%$ of control levels, respectively (Fig. $2 b$ ). In addition, because shLASP2a and shLASP2b were designed based upon previously validated siRNAs against LASP2 (Bliss et al., 2013), we are confident that they knock down endogenous LASP2.

Next, to determine the role of LASP1 in spine development, we transfected day in vitro 10 (DIV10) neurons with shRNAs and imaged them at DIV21 (Fig. 2c). This time period is critical for the formation of dendritic spines as well as their transition from a thin filopodial-like morphology to a mature mushroom-like shape (Yoshihara et al., 2009). We detected a significant reduction in the overall density of dendritic protrusions as well as a slight reduction in spine width in LASP1-deficient neurons compared with controls (Fig. 2c). In addition, we observed a signifi-

\section{$\leftarrow$}

(Figure legend continued.) $\quad p<0.0001$ ), total dendritic length (Kruskal-Wallis test: $p=$ $0.3215, \mathrm{H}=2.269, \mathrm{df}=2$; Dunn's test: GFP vs GFP-LASP1, $p>0.9999$; GFP vs GFP-LASP2, $p=0.4459$ ), and mean dendritic branch length (Kruskal-Wallis test: $p<0.0001, H=24.54$, $\mathrm{df}=2$; Dunn's test: GFP vs GFP-LASP1, $p>0.9999 ;$ GFP vs GFP-LASP2, $p<0.0001$ ). (b) Top left, images from DIV 21 hippocampal neurons expressing pSuper-mCherry, shLASP1a, or shLASP1b ( $n=49,41$, and 40, respectively). Top right, Sholl analysis demonstrates no change in dendritic complexity. Bottom, Quantification of the number of dendritic tips (Kruskal-Wallis test: $p=0.1683, \mathrm{H}=3.563, \mathrm{df}=2$; Dunn's test: $p$ Super vs shLASP1a, $p=0.5813$; $\mathrm{pS}$ uper vs shLASP1b, $p=0.6923$ ), total dendritic length (Kruskal-Wallis test: $p=0.2541, \mathrm{H}=2.74$, $\mathrm{df}=2$; Dunn's test: $p$ Super vs shLASP1a, $p=0.2394$; pSuper vs shLASP1b, $p>0.9999$ ), and the mean dendritic branch length (Kruskal-Wallis test: $p=0.7207, \mathrm{H}=0.6551$, df $=2$; Dunn's test: $p$ Super vs shLASP1a, $p=0.8992 ;$ pSuper vs shLASP1b, $p=0>0.9999)$. c, Top left, Images of pSuper, shLASP2a, or shLASP2b-expressing neurons ( $n=48,46$, and 45 , respectively). Top right, Changes in dendritic complexity are illustrated by Sholl analysis. Bottom, Graphs of dendritic tip numbers (Kruskal-Wallis test: $p<0.0001, \mathrm{H}=74.22$, df $=2$; Dunn's test: pSuper vs shLASP2a, $p<0.0001$; pSuper vs shLASP2b, $p<0.0001$ ), total dendritic length (Kruskal-Wallis test: $p<0.0001, \mathrm{H}=59.86, \mathrm{df}=2$; Dunn's test: pSuper vs shLASP2a, $p<$ 0.0001 ; pSuper vs shLASP2b, $p<0.0001$ ), and the mean dendritic branch length (KruskalWallis test: $p=0.0022, \mathrm{H}=12.2, \mathrm{df}=2$; Dunn's test: $p$ Super vs shLASP2a, $p=0.0021$; $\mathrm{pSuper}$ vs shLASP2b, $p=0.0415$ ). All graphs represent data collected from three independent experiments. Scale bars, $50 \mu \mathrm{m}$. Error bars for Sholl analysis represent $95 \% \mathrm{Cl}$, otherwise error bars represent the SEM. ${ }^{*} p \leq 0.05,{ }^{* *} p \leq 0.01,{ }^{* * *} p \leq 0.001$ by Kruskal-Wallis test with Dunn's post hoc test. cant increase in the proportion of spines exhibiting a filopodiallike morphology (Fig. 2c). This suggests that LASP1 may be required for spine development. Next, we examined the effects of GFP-LASP1 overexpression on spine development. We found that exogenous LASP1 expression did not detectably affect either spine density or morphology (Fig. $2 d$ ). Together, this suggests that there may be a minimum level of LASP1 expression required for normal spine development.

The effects of LASP2 knockdown on spine development appear to be much more substantial than LASP1 knockdown. Both the overall density and width of dendritic spines are markedly reduced, resulting in an approximately threefold increase in the proportion of filopodial-like protrusions (Fig. 2e). Conversely, the overexpression of GFP-LASP 2 induced a $\sim 20 \%$ increase in dendritic spine density and a $\sim 33 \%$ increase in spine width (Fig. $2 f$ ). We detected no significant change in the percentage of filopodial-like spines (Fig. $2 f$ ), likely due to the already low percentage of filopodial-like spines at DIV21. Together, these results indicate an important role for LASP proteins (both LASP1 and LASP2) in spine development. The loss/gain-of-function results of LASP2 further suggest that LASP2 may regulate spine development bidirectionally.

Because both spine density and spine width are reduced in LASP-depleted neurons, this suggested that synapse formation could also be affected. To investigate this, we examined the number of postsynaptic PSD-95-GFP puncta that cocluster with presynaptic SV2 puncta. The vast majority of dendritic spines from control pSuper neurons contain PSD-95-GFP signals that overlap with SV2 (Fig. 3a). We found that LASP1 knock-down neurons exhibited no significant change in synaptic density, compared with control neurons (Fig. $3 a$ ). On the other hand, LASP2-depleted neurons displayed a striking reduction in synaptic density (Fig. 3a). These findings suggest that LASP2, but not LASP1, plays a prominent role in synapse formation. To investigate this more directly, we performed whole-cell voltage-clamp recordings of hippocampal neurons at DIV19-21. To account for differences in neuronal density and circuit formation, we used nontransfected neurons as a control. pSuper neurons exhibited mEPSCs with similar amplitude and frequency as nontransfected controls (Fig. 3b). Likewise, we found that knockdown of LASP1 did not significantly affect either the amplitude or frequency of mEPSCs, compared with control neurons (Fig. 36 ). In contrast, LASP2 knockdown caused a large significant reduction in the frequency of mEPSCs, but only a slight decrease in mEPSC amplitude (Fig. 3b). This further suggests that the knockdown of LASP2 decreases the overall number of excitatory synapses per neuron.

\section{Regulation of dendritic arborization by LASPs}

Changes in synaptic activity in vitro and in vivo have previously been shown to affect the length and complexity of dendritic arbors (Sin et al., 2002; Peng et al., 2009; Cheadle and Biederer, 2014). To test whether LASP1 and LASP2 play a role in dendritic development, we first analyzed the dendritic arbors of neurons overexpressing GFP, GFP-LASP1, or GFP-LASP2 (Fig. 4a). We did not observe any differences in the number, total length, and complexity of dendritic branches in neurons overexpressing GFP-LASP1, compared with GFP controls (Fig. 4a). In contrast, we found that GFP-LASP2 expression increased the number of dendritic branch tips by $\sim 52 \%$ and decreased the mean branch length by $\sim 25 \%$, relative to control neurons (Fig. $4 a$ ). Furthermore, Sholl analysis revealed that GFP-LASP2 expression sharply increased the complexity of the dendritic arbor (number of in- 
a

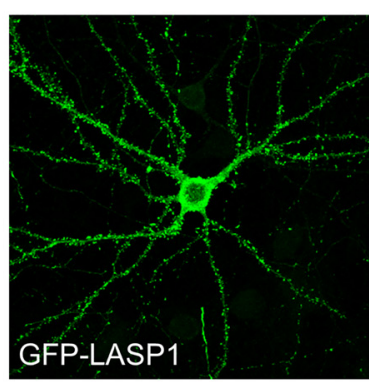

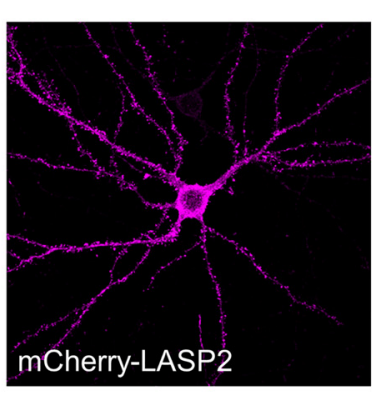

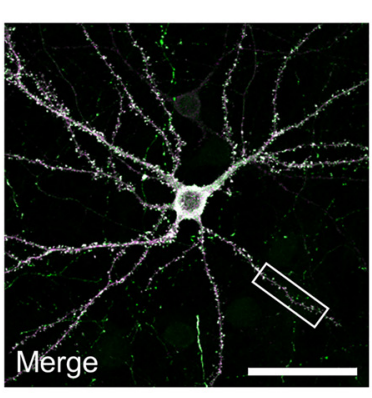

b
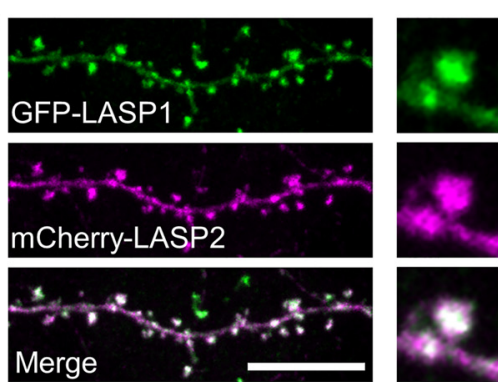

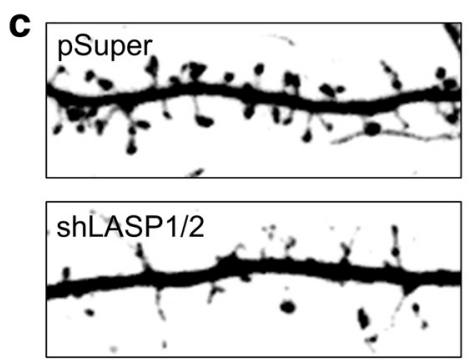

d

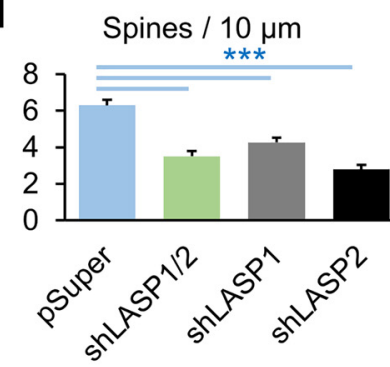

e
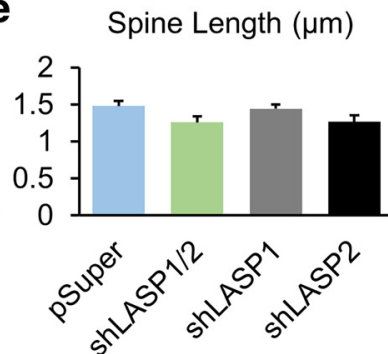

f

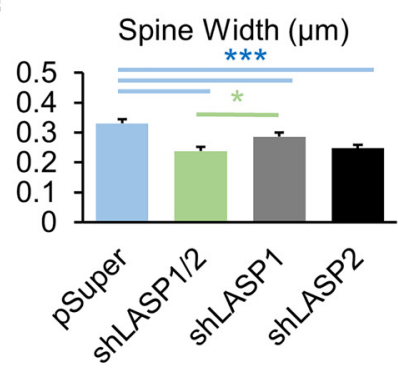

g

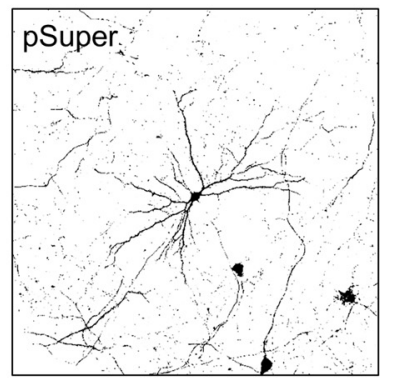

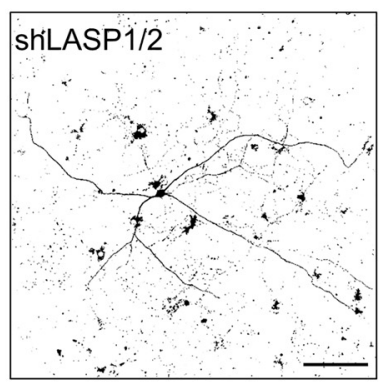

h
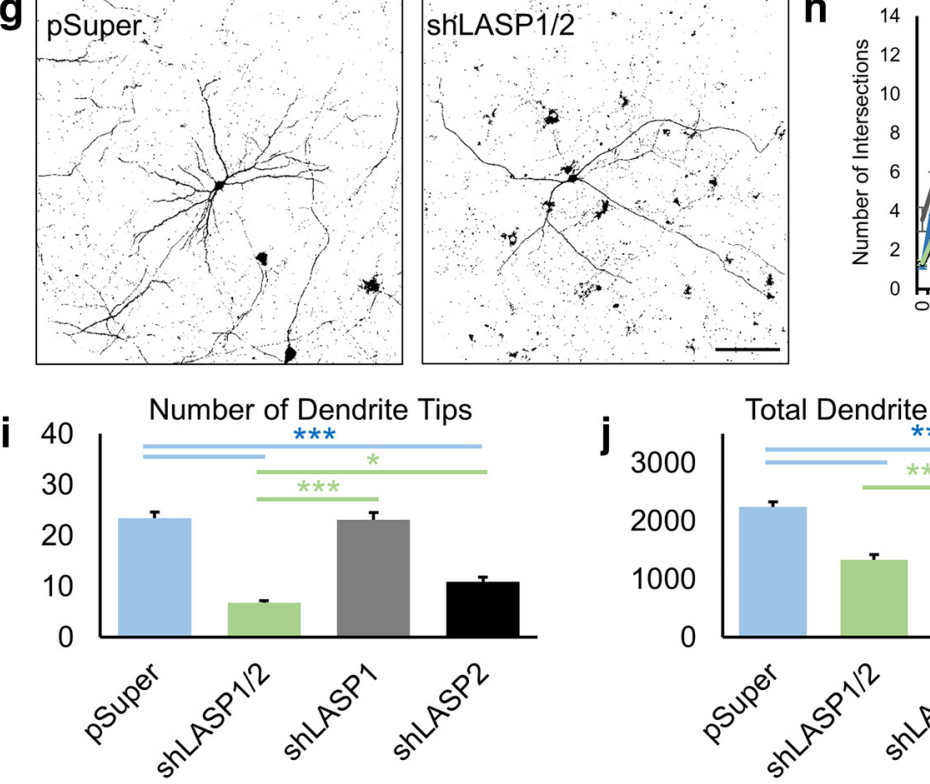

Total Dendrite Length $(\mu \mathrm{m})$

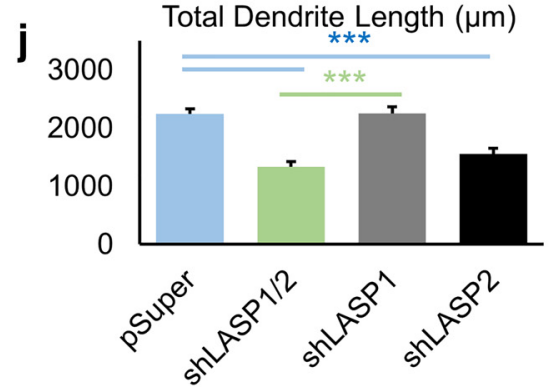

Sholl Analysis

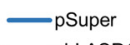

shLASP1

- ShLASP2

Figure 5. LASP proteins have redundant functions in spines, but not in dendritic arbors. $\boldsymbol{a}$, Images showing colocalization of GFP-LASP1 (green) and mCherry-LASP2 (magenta) in DIV21 hippocampal neurons. Scale bar, $50 \mu \mathrm{m} . \boldsymbol{b}$, Left, Inset of boxed region from neuron (a); right, inset of spine from left panel. Scale bar, $5 \mu \mathrm{m}$. $\boldsymbol{c}$, Representative images of dendritic spines from DIV21 neurons transfected with pSuper, or both shLASP1a and shLASP2a (shLASP1/2). Scale bar, $5 \mu \mathrm{m}$.d, Quantification of spine density (Kruskal-Wallis test: $p<0.0001, \mathrm{H}=45.46, \mathrm{df}=3 ;$ Dunn's test: pSuper vs shLASP1/2, $p<0.0001 ;$ pSuper vs shLASP1, $p=0.0024$; pSuper vs shLASP2, $p<0.0001$ ). , Quantification of spine length (Kruskal-Wallis test: $p=0.0528, \mathrm{H}=7.694, \mathrm{df}=3 ;$ Dunn's test: $p S$ uper vs shLASP1/2, $p=2707 ;$ PSuper vs shLASP1, $p>0.9999 ;$ SSuper vs shLASP2, $p=0.1478$ ). $f$, Quantification of spine width (Kruskal-Wallis test: $p<0.0001, \mathrm{H}=36.05, \mathrm{df}=3 ;$ Dunn's test: $p$ Super vs shLASP1/2, $p<0.0001 ;$ PSuper vs shLASP1, $p=0.0243$; pSuper vs shLASP2, $p<0.0001$; shLASP1/2 vs shLASP1, $p=0.0304 ; n=519$ spines from 18 cells for $p$ Super, 325 spines from 18 cells for shLASP1/2, 342 spines from 18 cells for shLASP1a, and 258 spines from 18 cells for shLASP2a). $g$, Representative images of DIV21 mCherry-expressing hippocampal neurons transfected at DIV11 with pSuper or shLASP1/2. Scale bar, $50 \mu \mathrm{m}$. $\boldsymbol{h}$, Sholl analysis for neurons expressing pSuper, shLASP1/2, shLASP1, and shLASP2. Error bars represent $95 \%$ CI. $\boldsymbol{i}$, Quantification of number of dendritic tips (one-way ANOVA: $p<0.0001, F_{(3,195)}=77.26$; Tukey's test: $p$ Super vs shLASP1/2, $p<0.0001 ; p S u p e r$ vs shLASP1, $p=0.9959 ; p S u p e r$ vs shLASP2, $p<0.0001$; shLASP1/2 vs shLASP1, $p<0.0001$; shLASP1/2 vs shLASP2, $p=0.0124)$.j, Quantification of total dendritic length (one-way AN0VA: $p<0.0001, F_{(3,195)}=24.6 ;$ Tukey's test: $p S$ uper vs shLASP1/2, $p<0.0001$; $p S$ uper vs shLASP1, $p=0.9999$; $p S$ uper vs shLASP2, $p<0.0001$; shLASP1/2 vs shLASP1, $p<0.0001$; shLASP1/2 vs shLASP2, $p=0.359)$. $\boldsymbol{k}$, Quantification of mean dendritic branch length (one-way ANOVA: $p<0.0001, F_{(3,195)}=31.74 ;$ Tukey's test: $p S$ uper vs shLASP1/2, $p<0.0001 ; p S$ uper vs shLASP1, $p=0.9958 ; p S u p e r$ vs shLASP2, $p<0.0001 ;$ shLASP1/2 vs shLASP1, $p<$ $0.0001 ;$ shLASP1/2 vs shLASP2, $p=0.0053 ; n=48$ cells/construct). All graphs represent data collected from three independent experiments. Error bars represent the SEM. ${ }^{*} p \leq 0.05,{ }^{*} p \leq 0.01$, ${ }^{* * *} p \leq 0.001$ by Kruskal-Wallis test with Dunn's post hoc test, or by one-way ANOVA with Tukey's multiple-comparisons test, and Shapiro-Wilk test for normality.

tersections per $10 \mu \mathrm{m}$ concentric ring) between 20 and $100 \mu \mathrm{m}$ radii from the soma, compared with both control and GFPLASP1 cells (Fig. 4a). This suggests that LASP2, but not LASP1, promotes dendritic arbor complexity.

To confirm this hypothesis, we knocked down endogenous LASP1 and LASP2, and examined their effects on arbor develop- ment. We found that the loss of LASP1 had no detectable effect on dendritic arbor formation (Fig. 4b). The knockdown of LASP2 on the other hand, resulted in large reductions in dendritic arbor complexity (Fig. 4c). Sholl analysis revealed decreases in the number of dendritic intersections observed between 20 and 220 $\mu \mathrm{m}$ radii (Fig. $4 c$ ). In addition, we found significant reductions in 
a
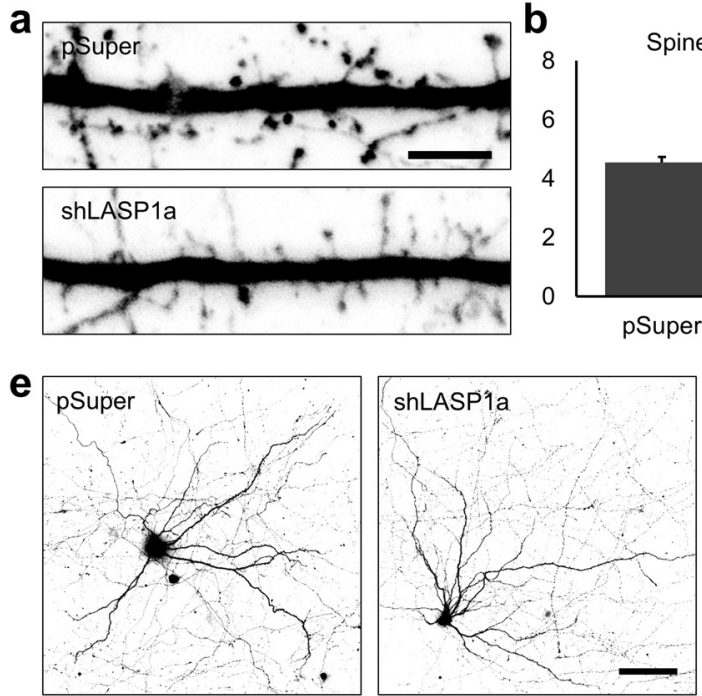

h
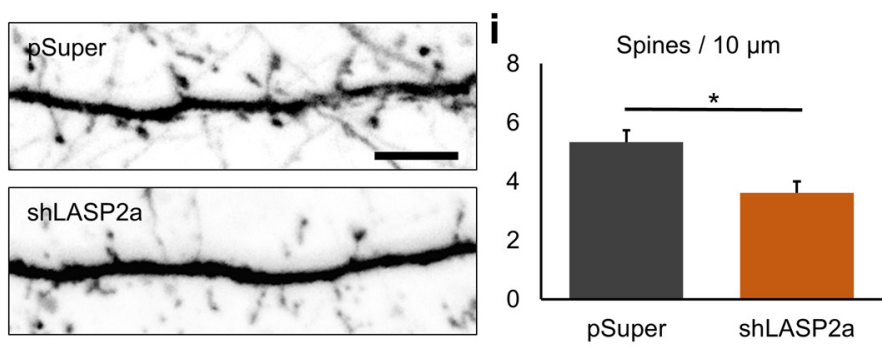

C

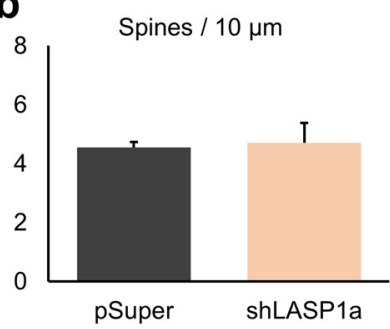

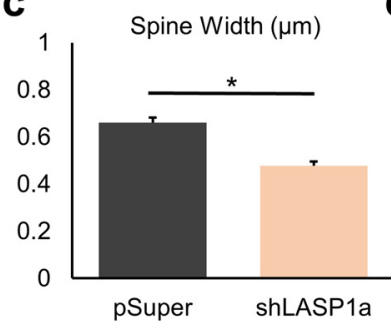

d

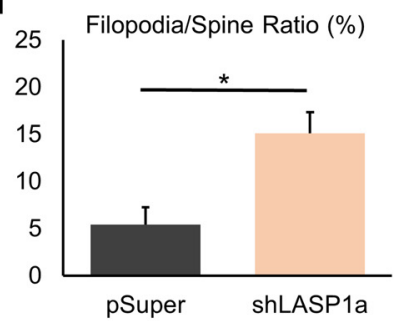

f
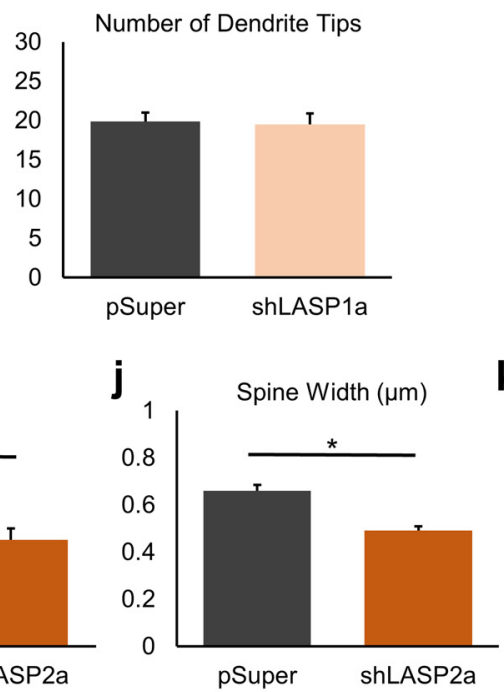

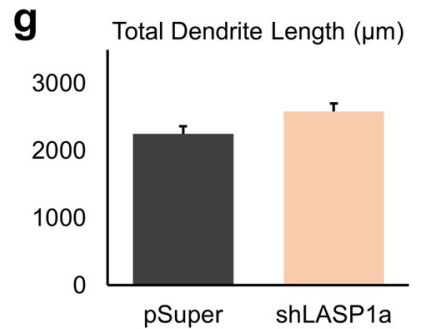

k
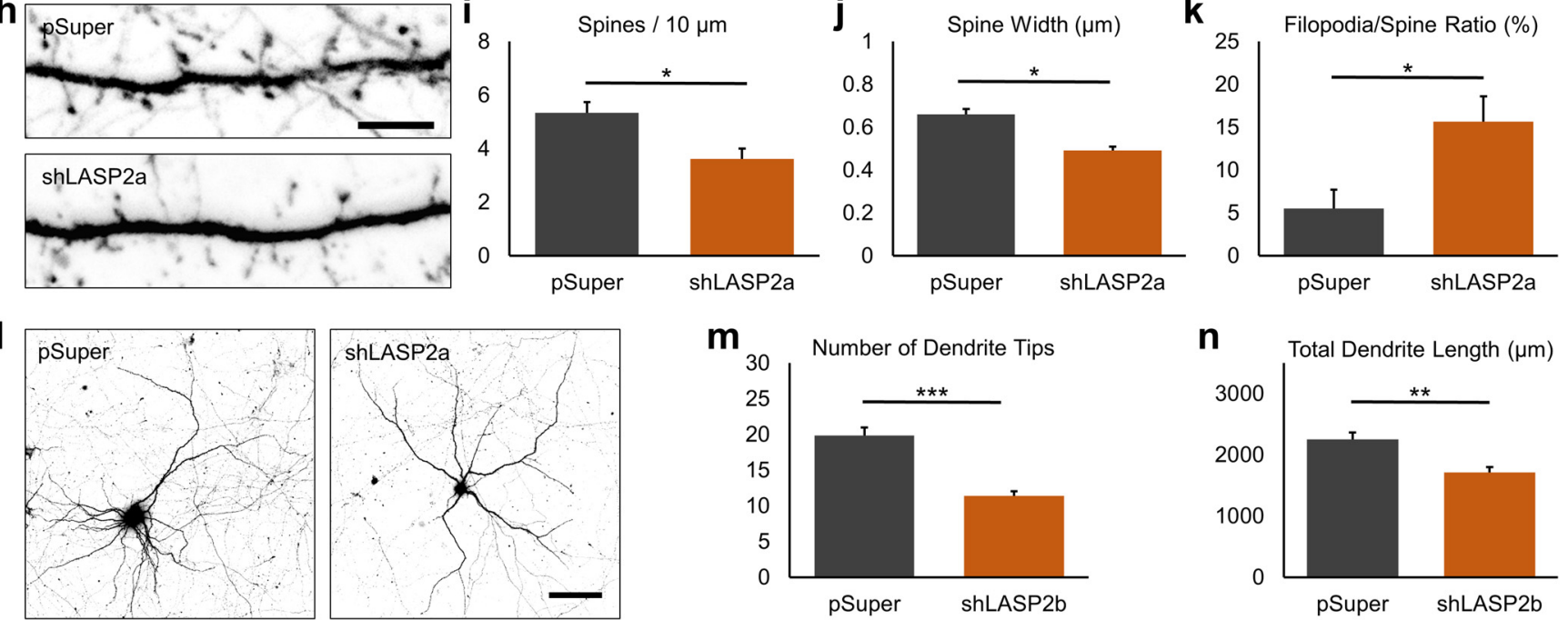

n

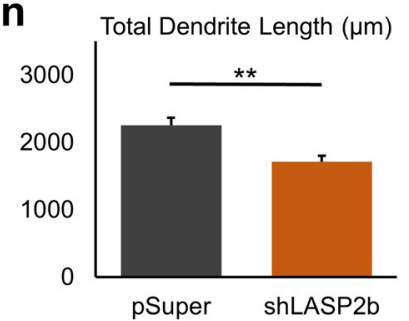

Figure 6. LASP2 regulates dendritic spine and arbor stabilization in mature neurons. $\boldsymbol{a}$, Hippocampal neurons were transfected at DIV15, and spines were imaged at DIV21. Scale bar, $5 \mu$ m. $\boldsymbol{b}-\boldsymbol{d}$, Graphs of spine density ( $\boldsymbol{b}$; Mann-Whitney test: $p=0.801, \mathrm{U}=79$ ), spine width ( $\boldsymbol{c}$; Mann-Whitney test: $p<0.0001, \mathrm{U}=10)$, and percentage of spines with filopodial-like morphology ( $\boldsymbol{d}$; Mann-Whitney test: $p=0.0015, \mathrm{U}=25 ; n=489$ spines from 13 cells for $p S$ uper, and 609 spines from 13 cells for shLASP1a). $e$, Images of DIV21 neurons expressing pSuper-mCherry or shLASP1a. $\boldsymbol{f}, \boldsymbol{g}$, Quantification of the number of dendritic tips ( $\boldsymbol{f}$; unpaired $t$ test: $p=0.8422, t=0.1998, \mathrm{df}=66)$ and total dendritic length $(\boldsymbol{g}$; unpaired $t$ test: $p=0.0897, t=1.722, \mathrm{df}=66 ; n=34$ cells/construct). $\boldsymbol{h}$, Images of spines from neurons transfected with pSuper or shLASP2a at DIV15 and imaged at DIV21. $\boldsymbol{i}-\boldsymbol{k}$, Graphs of spine density $(\boldsymbol{i} ;$ Mann-Whitney test: $p=0.0102, \mathrm{U}=35)$, spine width (; Mann-Whitney test: $p<0.0001, \mathrm{U}=10$ ), and percentage of spines with filopodial-like morphology $(\boldsymbol{k} ;$ Mann-Whitney test: $p=0.0051, \mathrm{U}=31 ; n=552$ spines from 13 cells for pSuper, and 489 spines from 13 cells for shLASP2a). I, Images of DIV21 neurons expressing pSuper-mCherry or shLASP2a. $\boldsymbol{m}, \boldsymbol{n}$, Graphs of number of dendritic tips ( $\boldsymbol{m}$; unpaired $t$ test: $p<0.0001$, $t=6.505, \mathrm{df}=67$ ) and total dendritic length ( $\boldsymbol{n}$; unpaired $t$ test: $p<0.0001, t=4.396, \mathrm{df}=67 ; n=34$ and 35 cells, respectively). Scale bars: $\boldsymbol{a}, \boldsymbol{h}, 5 \mu \mathrm{m} ; \boldsymbol{e}, \boldsymbol{l}, 50 \mu \mathrm{m}$. All graphs represent data collected from three independent experiments. All error bars represent the SEM. ${ }^{*} p \leq 0.05,{ }^{* *} p \leq 0.01,{ }^{* * *} p \leq 0.001$ by Mann-Whitney test and unpaired $t$ test.

both the number of dendritic tips and total dendritic length in LASP2 knock-down neurons relative to pSuper control neurons (Fig. 4c). This corresponds with the LASP2 overexpression phenotype and indicates that LASP2 promotes dendritic arbor complexity and growth.

Next, we wanted to determine whether LASP1 and LASP2 have overlapping or distinct functions during neuronal development. We first examined their subcellular localization in live hippocampal neurons at DIV21 using GFP-tagged LASP1 and mCherry-tagged-LASP2 (Fig. $5 a$ ). Both proteins are highly colocalized, particularly in spines (Fig. $5 b$ ). Next, we knocked down LASP1 and LASP2 singly and simultaneously (shLASP1/2) at DIV11 and analyzed dendritic spines at DIV21 (Fig. 5c). We found that the density and morphology of spines from shLASP1/2 neurons were essentially indistinguishable from LASP1 and LASP2 individual knock-down neurons (Fig. $5 d-f$ ). However, we detected significant decreases in overall dendritic complexity, branch number, and total dendritic length in double knock-down neurons compared with pSuper control neurons and LASP1 single knock-down neurons (Fig. $5 g-k$ ). Interestingly, we detected an additional small but significant reduction in the number of dendritic tips and an increase in mean branch length in shLASP1/2 cells, compared with LASP2 single knock-down neurons (Fig. $5 i-k$ ). This suggests that LASP1 may play some small overlapping role in dendritic arbor development, but that this minor function can be compensated for by LASP2.

One concern regarding the effects of LASP2 knockdown on dendritic spines is that these effects may be secondary to the defects in dendritic arbor formation. To address this, we performed shRNA knockdown of LASP1 and LASP2 starting at 


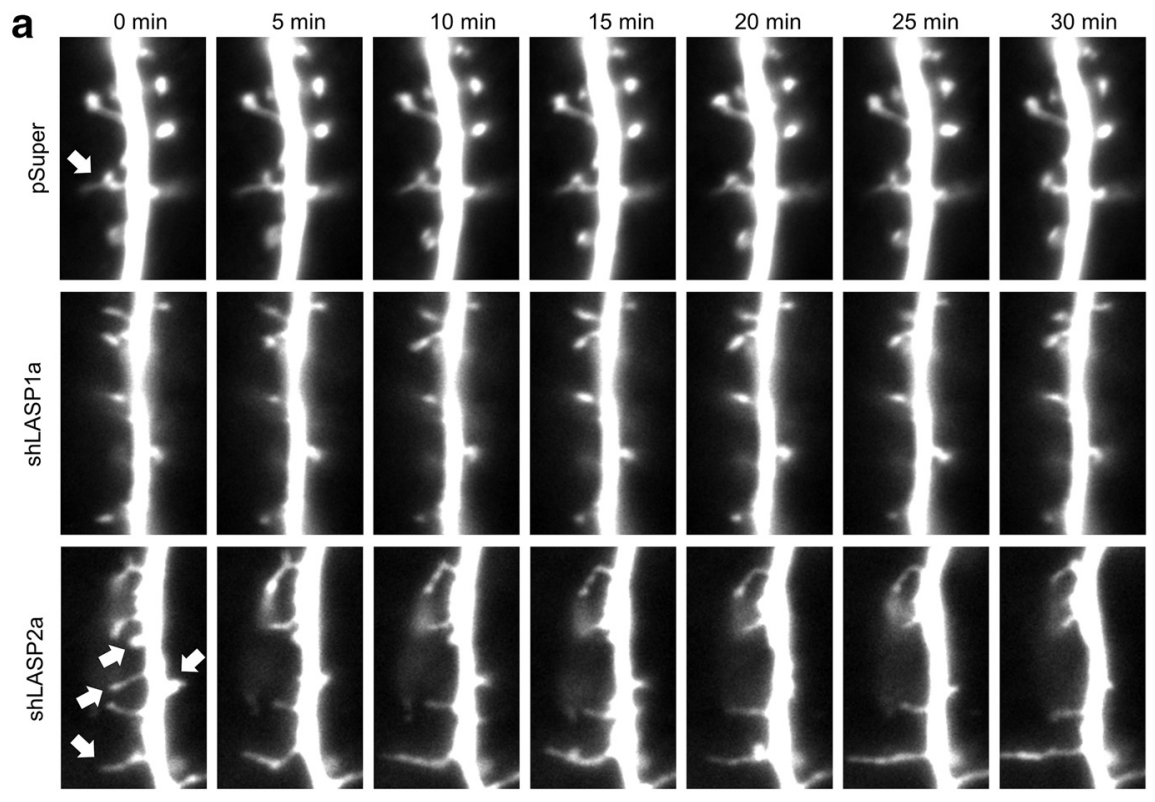

b
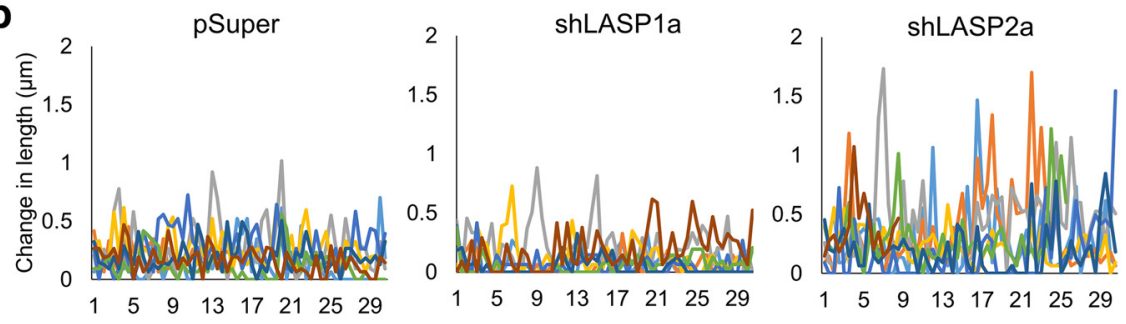

C

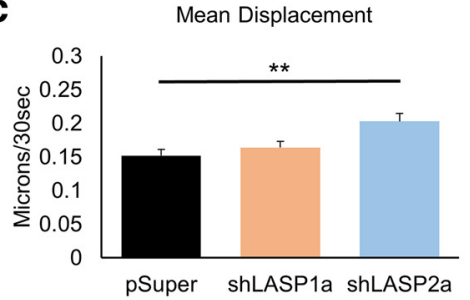

d

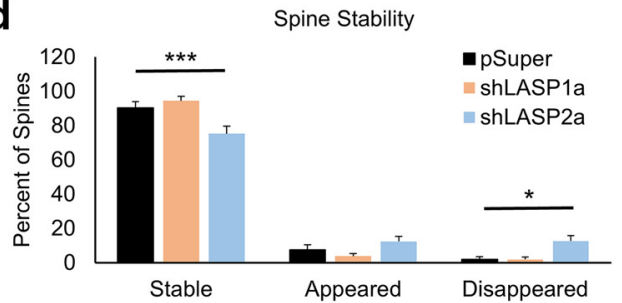

Figure 7. LASP2 knockdown destabilizes dendritic spines. $\boldsymbol{a}$, Representative time-lapse images show spines from neurons expressing pSuper-mCherry, shLASP1a, or shLASP2a, imaged every $30 \mathrm{~s}$ for $30 \mathrm{~min}$. Arrows indicate dynamic spines. $\boldsymbol{b}$, Sample traces show changes in spine length over the recording period, with each trace representing an individual spine. $c$, Graph shows mean displacement of spines length (one-way ANOVA: $p=0.0010, F_{(2,370)}=7.019$; Tukey's test: $p S$ upervs shLASP1, $p=0.6960$; pSuper vs shLASP2, $p=0.0010$ ). (d) Graph shows the relative stability of spines over the imaging period length (two-way ANOVA: $p<0.0001, F_{(4,72)}=9.844$; Dunnett's test: stable, $p S$ uper vs shLASP1, $p=0.5138$; stable, pSuper vs shLASP2, $p=0.0006$; appeared, pSuper vs shLASP1, $p=0.5323$; appeared, pSuper vs shLASP2, $p=0.3989$; disappeared, pSuper vs shLASP1, $p=$ 0.9994 ; disappeared, pSuper vs shLASP2, $p=0.02 ; n=125$ spines from 9 cells for $p$ Super, 104 spines from 9 cells for shLASP1a, and 111 spines from 9 cells for shLASP2a). All graphs represent data collected from three independent experiments. Error bars represent the SEM. ${ }^{*} p \leq 0.05,{ }^{* *} p \leq 0.01,{ }^{* * *} p \leq 0.001$ by one-way ANOVA with Tukey's post hoc test or by two-way ANOVA with Dunnett's post hoc test.

DIV15 and imaging on DIV21. By DIV15, hippocampal neurons in culture have mostly completed dendritic growth and arborization, synapse formation has peaked, and the majority of spines have formed but not matured (Ziv and Smith, 1996; Grabrucker et al., 2009). Therefore, the transfection of DIV15 hippocampal neurons should minimize the effects on early dendrite development and synaptogenesis. We found that LASP1 knockdown by DIV15 transfection did not affect spine density relative to control neurons, but did reduce spine width and increased the proportion of filopodial-like spines (Fig. $6 a-d$ ). This suggests that LASP1 is in fact involved in spine development, and more specifically, it may play a role in the conversion of filopodia into spines.
We also examined the dendritic arbors of these DIV15 transfected neurons and could not detect any effects on dendritic arbor complexity or size (Fig. $6 e-g$ ).

In contrast, we observed significant reductions in both spine density and spine width in LASP2 knock-down neurons, as well as a corresponding increase in the proportion of filopodia-like spines relative to control neurons (Fig. $6 h-k$ ). The reduction in spine density is particularly interesting, as it suggests that LASP2 may be required for the stabilization or maintenance of spines, rather than the conversion of filopodia to spines. Strikingly, LASP2 knockdown also caused reductions in dendritic arbor complexity as assessed by Sholl analysis (Fig. 6l$n$ ). The number of dendritic branch tips and the total dendritic length were also significantly reduced in these older LASP2 knockdown neurons (Fig. 6l-n). Dendritic arbors are largely stabilized by DIV15 and do not normally undergo retractions after this point (Grabrucker et al., 2009; Koleske, 2013). Therefore, these data suggest that LASP2 may regulate the stability of dendritic branches.

\section{LASP2 regulates the stability of dendritic protrusions}

Spine stabilization is marked by a morphological transition from filopodial-like spines to mushroom-shaped spines (Lin and Koleske, 2010; Berry and Nedivi, 2017). The process of spine stabilization is particularly important because it corresponds to the consolidation of newly acquired information into long-term memory (Berry and Nedivi, 2017). To directly determine whether LASP2 plays a role in spine stabilization, we knocked down LASP2 and performed live imaging of dendritic spines every $30 \mathrm{~s}$ for $30 \mathrm{~min}$ (Fig. 7a). We then tracked the movement of spine tips and analyzed the distance each spine tip moved (displacement) per frame. Knocking down LASP2 leads to significantly more dynamic dendritic spines, compared with either pSupercontrol or LASP1-depleted neurons (Fig. $7 b, c)$. In addition, while spines from control and LASP1 knock-down neurons were $90 \%$ and $94 \%$ stable during the imaging time frame, respectively, only $75 \%$ of LASP2 knock-down spines were stable (Fig. 7d). These data indicate that LASP2 is in fact required for the stabilization of dendritic spines. It also provides further evidence that LASP1 is involved in spine development, rather than spine stabilization.

We wondered whether the knockdown of LASP2 affects the growth or the stability of dendritic arbors, similar to its role in spines. To test this, we used live imaging of individual LASP1 and LASP2 knock-down neurons every 15 min over 18 h (Fig. 8a). By tracking the movement of individual dendritic branch tips, we 
a

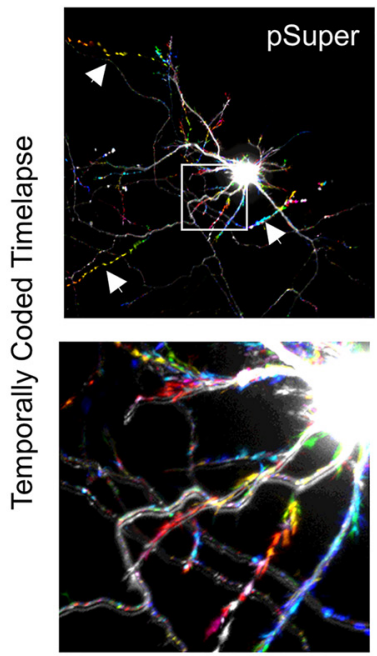

b

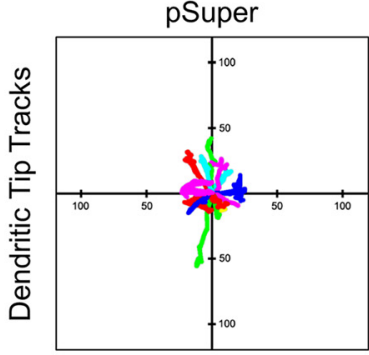

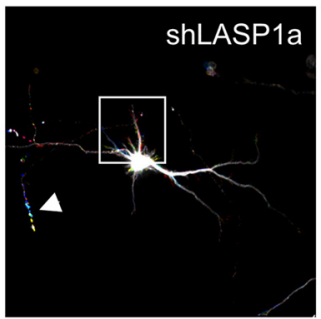

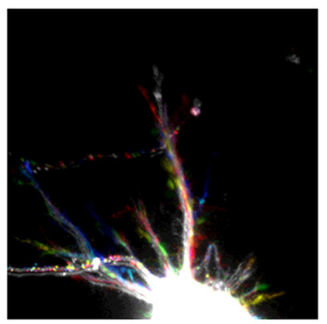

shLASP1a

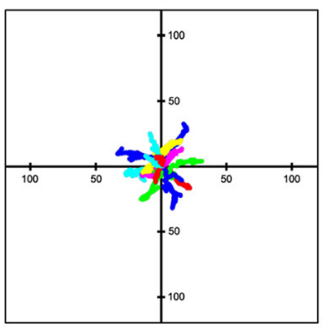

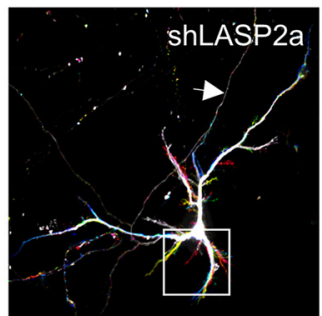

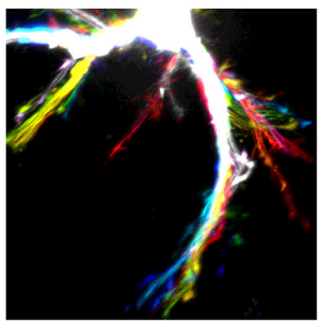

shLASP2a

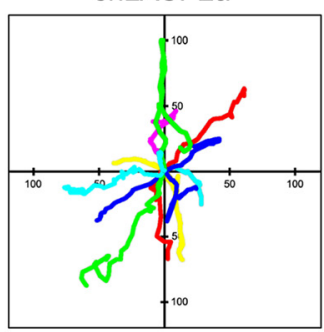

C

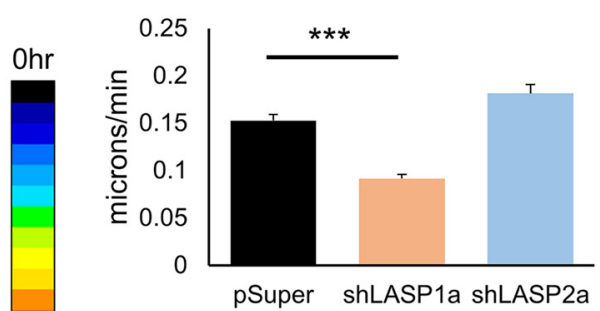

e

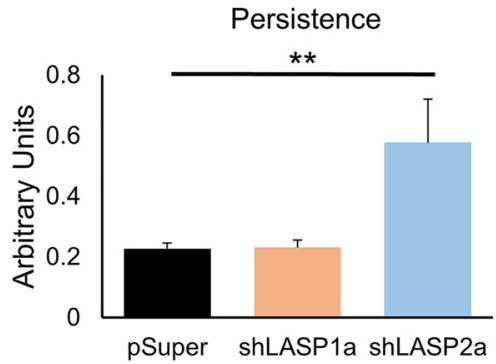

f

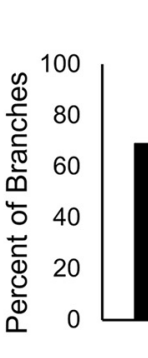

$* * *$

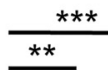

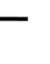
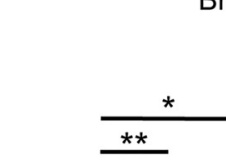

Branch Stability

d

Mean Displacement

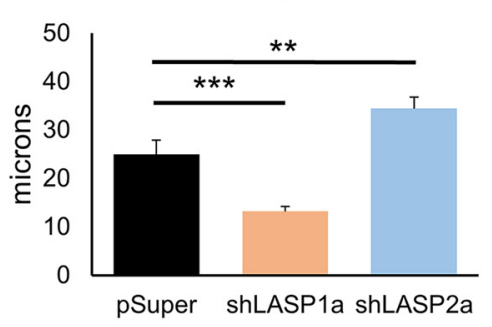

- pSuper

shLASP1a

- shLASP2a

Figure 8. LASP2 knockdown destabilizes dendritic arbors. $\boldsymbol{a}$, Top, Images show individual hippocampal neurons temporally color coded to illustrate branch motility over time (scale shows correlation of image time to color). Neurons were transfected with pSuper, shLASP1a, or shLASP2a constructs on DIV5, and imaged every 15 min for $18 \mathrm{~h}$ starting on DIV11. Arrows indicate axons. Bottom, Insets from boxes in corresponding images from top row. $\boldsymbol{b}$, Rose plots show representative traces of individual dendritic branch tip movements from the corresponding neuron above. $\boldsymbol{c}-\boldsymbol{f}$, Histograms show mean velocity of branch tips (c; Kruskal-Wallis test: $p<0.0001, \mathrm{H}=85.51$, df $=2$; Dunn's test: $\mathrm{pSuper}$ vs shLASP1, $p<0.0001 ; \mathrm{pS}$ uper vs shLASP2, $p=0.3719$ ), mean tip displacement (d; Kruskal-Wallis test: $p<0.0001, \mathrm{H}=61.03$, df $=2$; Dunn's test: pSuper vs shLASP1, $p<0.0001 ; \mathrm{pSuper}$ vs shLASP2, $p=0.0014$ ), tip persistence, which we defined as displacement over the total distance traveled ( $\boldsymbol{e}$; Kruskal-Wallis test: $p<0.0001, \mathrm{H}=23.68, \mathrm{df}=2 ;$ Dunn's test: $\mathrm{pSuper}$ vs shLASP1, $p=0.8126 ; \mathrm{pS}$ uper vs shLASP2, $p=0.003$ ), and the relative stability of dendritic branches ( $f$; two-way ANOVA: $p<0.0001, F_{(6,68)}=28.12$; Dunnett's: stable, pSuper vs shLASP1, $p=0.0010$; stable, $p S$ uper vs shLASP2, $p=0.0001$; appeared, $p S$ uper vs shLASP1, $p=0.0012$; appeared, pSuper vs shLASP2, $p=0.0412$; disappeared, pSuper vs shLASP1, $p=0.9952 ;$ disappeared, $p$ Super vs shLASP2, $p=0.0210 ;$ appeared/disappeared, $p$ Super vs shLASP1, $p=0.9878$; appeared/disappeared, $p S$ uper vs shLASP2, $p=0.1509 ; n=7$ cells/construct). All graphs represent data collected from three independent experiments. Error bars represent the SEM. ${ }^{*} p \leq 0.05,{ }^{* *} p \leq 0.01,{ }^{* *} p \leq 0.001$ by Kruskal-Wallis test with Dunn's test post hoc test or two-way ANOVA with Tukey's post hoc test.

were able to detect major changes in the motility of dendritic branches after LASP2 knockdown (Fig. 8b). For example, compared with pSuper controls, LASP2 knock-down dendrites displayed significant increases in both mean tip displacement and tip persistence, which we define as the ratio of net displacement to the total distance traveled (Fig. $8 c-e$ ). The percentage of dendritic branches that were stable during the observation period was also decreased by half in LASP2 knock-down neurons relative to controls. (Fig. $8 f$ ). This increase in less stable, more motile branches in LASP2 knock-down neurons is similar to the spine phenotype we observed. Altogether, it suggests that LASP2 is a major regulator of dendrite stability. In contrast, LASP1 knock-down branches were slightly more stable and slightly less motile, as assessed by small but significant reductions in mean displace- ment and velocity as well as an increase in the percentage of stable branches (Fig. $8 c-f$ ). These data suggest that the reduction in dendritic complexity observed in LASP2 knock-down neurons is, in fact, caused by a decrease in branch stability rather than an effect on branch growth.

The actin-binding domains of LASP2 are required for its ability to stabilize dendritic spines and dendritic arbors LASP2 has previously been shown to bind and directly bundle F-actin (Zieseniss et al., 2008), similar to other nebulin family members. The N-terminal LIM domain and nebulin repeats of LASP2 cooperatively facilitate a direct interaction with F-actin, and are likely essential for its actin-bundling activity (Li et al., 2004; Lin et al., 2004; Nakagawa et al., 2009). We hypothesize that 
a

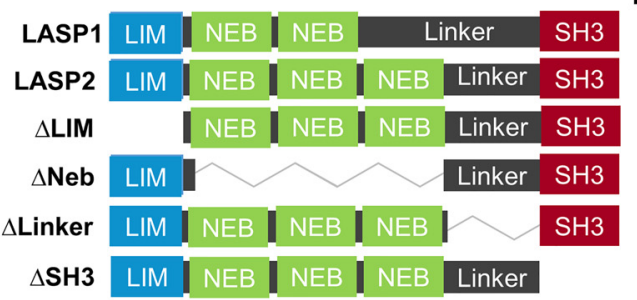

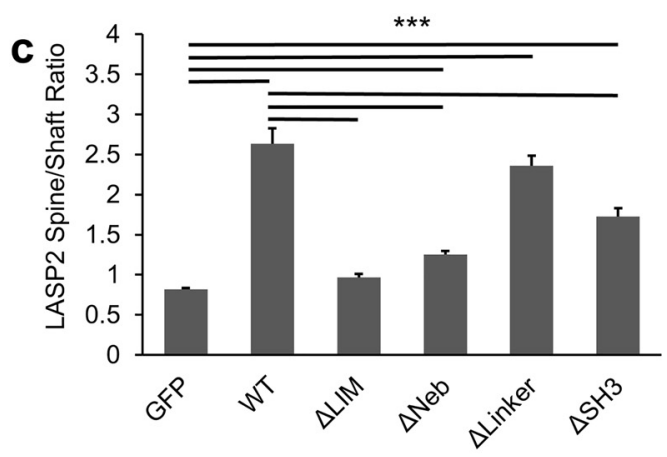

b GFP-LASP2
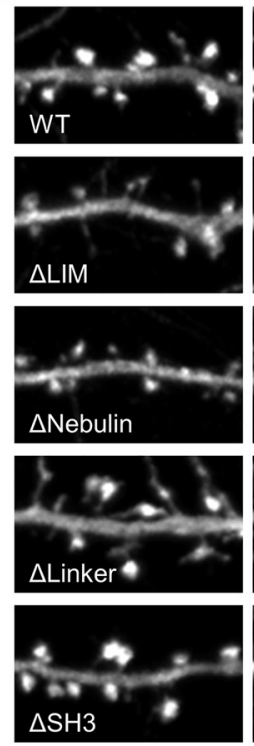
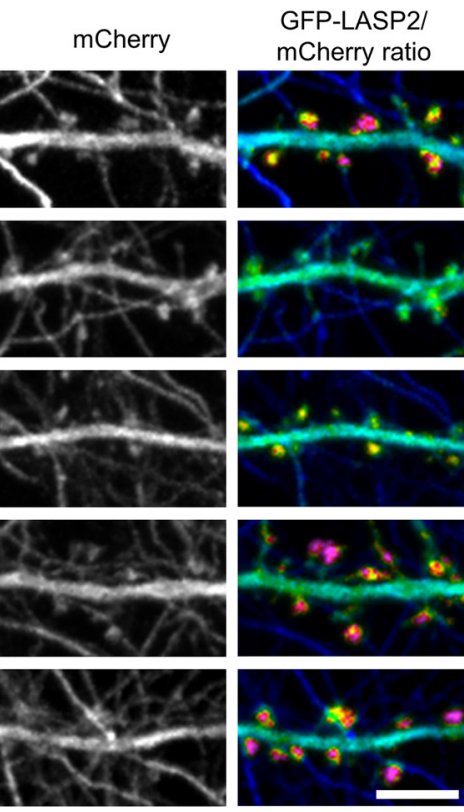

GFP-LASP1/

mCherry ratio

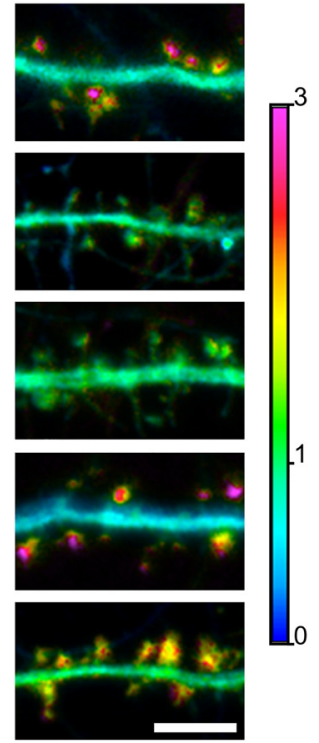

d
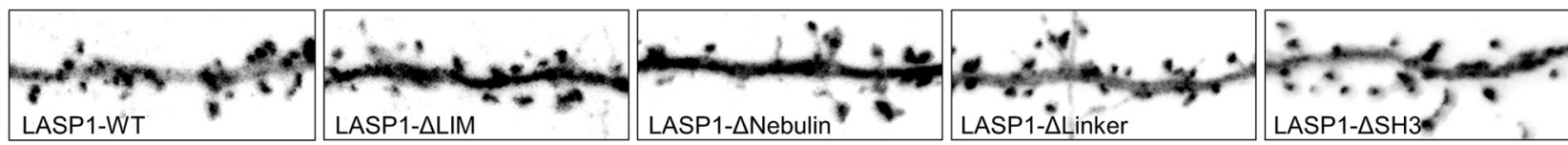

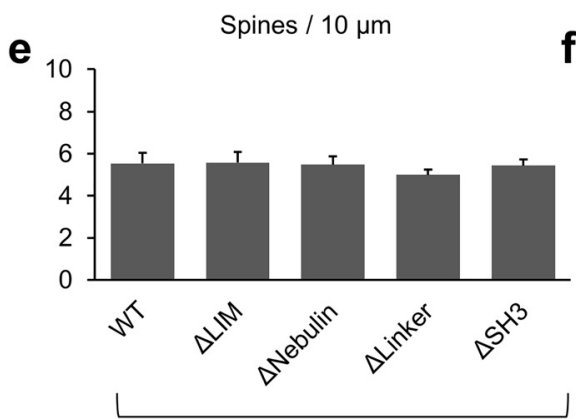

GFP-LASP1

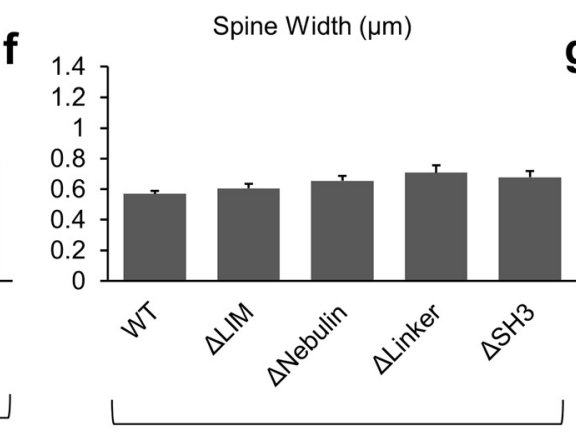

GFP-LASP1

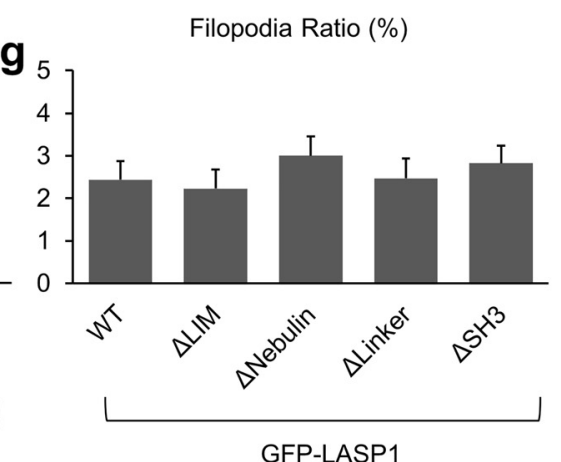

GFP-LASP1

h

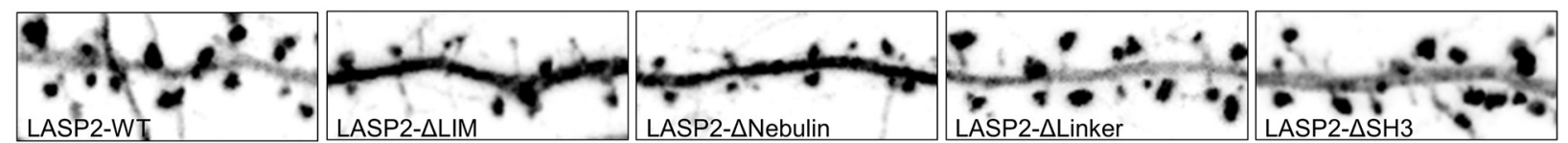

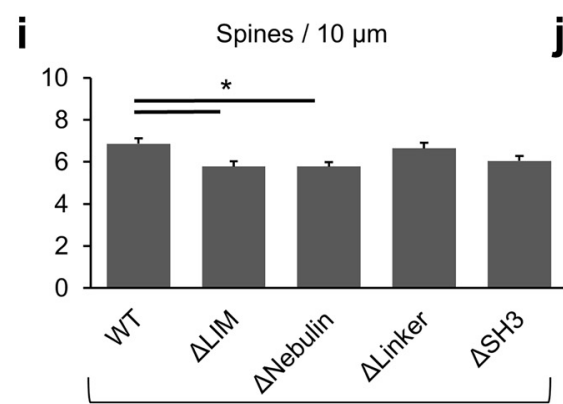

GFP-LASP2

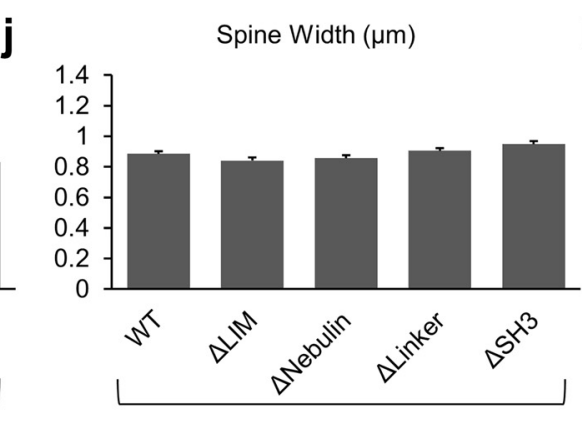

GFP-LASP2 k Filopodia Ratio (\%)

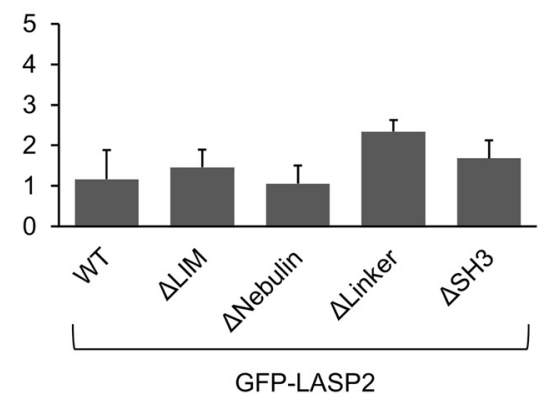

Figure 9. The actin-binding LIM domain and nebulin repeats of LASP2 are required for dendritic spine stabilization. $\boldsymbol{a}$, Domain organizations of LASP1, LASP2, and LASP2 domain deletion mutants. $\boldsymbol{b}$, Representative images showing the enrichment of GFP-LASP2 and GFP-LASP1 truncation mutants in dendritic spines. Neurons were cotransfected with soluble mCherry as a volume marker, and ratiometric images are shown to illustrate the relative enrichment of each mutant construct in spines. Scale bars, $5 \mu \mathrm{m}$. $c$, Graph of spine enrichment of GFP-LASP2 truncation mutants ( $n=144$ spines from 18 cells/construct). Fluorescent intensity in spines was normalized to the intensity of the adjacent dendritic shaft (one-way ANOVA: $p<0.0001$, (Figure legend continues.) 
LASP2-mediated actin bundling stabilizes dendritic spines and dendritic arbors. To test this, we wanted to determine the roles of these domains on spine and branch stability. Therefore, we engineered a series of LASP1 and LASP2 deletion mutants lacking the LIM domain ( $\Delta$ LIM), nebulin repeats ( $\Delta$ Nebulin), linker region ( $\Delta$ Linker), or C-terminal SH3 domain $(\Delta \mathrm{SH} 3$; Fig. $9 a)$. These GFP-tagged mutants were coexpressed with mCherry as a volume marker and were visualized as a ratio of GFP to mCherry (Fig. 9b). To quantify the enrichment of each deletion mutant in spine heads, we examined the H/S ratio (Fig. 9c). The LASP2$\Delta$ Linker mutant was highly enriched in spines, similar to wildtype GFP-LASP2. On the other hand, the LASP2- $\Delta$ LIM and LASP2- $\Delta$ Nebulin mutants were not enriched in spines, and are essentially indistinguishable from soluble GFP (Fig. 9c). Interestingly, the LASP2- $\Delta \mathrm{SH} 3$ mutant displayed an intermediate level of enrichment in spines (Fig. 9c). This suggests that LASP2 enrichment in spines is largely mediated by binding to F-actin via the LIM domain and nebulin repeats, although the SH3 domain does appear to play some role as well. We observed similar localization patterns when we expressed the corresponding LASP1 truncation mutants (Fig. 9b).

Next, we examined the effects of the LASP1 deletion mutants on dendritic spines and did not detect any changes in spine density or morphology, similar to WT-LASP1 expression (Fig. 9d-g). Similarly, spines from neurons overexpressing the GFP-LASP2$\Delta \mathrm{SH} 3$ and GFP-LASP2- $\Delta$ Linker mutants appeared indistinguishable from GFP-LASP2-WT-expressing cells (Fig. 9h-k), suggesting that neither domain is essential for regulating dendritic spine morphology. Conversely, we found that neurons expressing the GFP-LASP2- $\Delta$ LIM and GFP-LASP2- $\Delta$ Nebulin mutants had significantly lower spine density compared with GFP-LASP2-WT-expressing neurons (Fig. 9h-k). This suggests that the LASP2 LIM domain and nebulin repeats, which jointly mediate actin binding, are required for its function in spines.

\footnotetext{
$\leftarrow$

(Figure legend continued.) $\quad F_{(5,766)}=118.2$; Tukey's test: GFP vs WT, $p<0.0001$; GFP vs $\Delta$ LIM, $p<0.5789$; GFP vs $\Delta$ Nebulin, $p<0.0001$; GFP vs $\Delta$ Linker, $p<0.0001$; GFP vs $\Delta$ SH3, $p<0.0001$, WT vs $\Delta$ LIM, $p<0.0001$; WT vs $\Delta$ Nebulin, $p<0.0001$; WT vs $\Delta$ Linker, $p=$ 0.1971; WT vs $\Delta S H 3, p<0.0001)$. $\boldsymbol{d}$, Images of dendritic spines from neurons expressing GFP-tagged LASP1 truncation mutants. Scale bar, $5 \mu \mathrm{m}$. $\boldsymbol{e}-\boldsymbol{g}$, Effects of GFP-LASP1 mutants expression on dendritic spine density ( $\boldsymbol{e}$; Kruskal-Wallis test: $p=0.8762, \mathrm{H}=1.212, \mathrm{df}=4$; Dunn's test: WT vs $\Delta$ LIM, $p>0.9999$; WT vs $\Delta$ Nebulin, $p>0.9999$; WT vs $\Delta$ Linker, $p>$ 0.9999 ; WT vs $\Delta$ SH3, $p>0.9999$ ), spine width ( $\boldsymbol{f}$; Kruskal-Wallis test: $p=0.2206, \mathrm{H}=5.726$, $\mathrm{df}=4$; Dunn's test: WT vs $\Delta \mathrm{LIM}, p>0.9999 ;$ WT vs $\Delta$ Nebulin, $p=0.6370 ;$ WT vs $\Delta$ Linker, $p=0.2595$; WT vs $\Delta S H 3$, p0.3339), and percentage of spines with filopodial-like morphology ( ; Kruskal-Wallis test: $p=0.6403, \mathrm{H}=2.524 \mathrm{df}=4$; Dunn's test: WT vs $\Delta$ LIM, $p>0.9999$; WT vs $\Delta$ Nebulin, $p>0.9999 ;$ WT vs $\Delta$ Linker, $p>0.9999 ;$ WT vs $\Delta S H 3, p>0.9999 ; n=606$ spines from 25 cells for WT, 520 spines from 21 cells for $\Delta$ LIM, 458 spines from 21 cells for $\Delta$ Nebulin, 360 spines from 19 cells for $\Delta$ Linker, and 499 spines from 23 cells for $\Delta S H 3) . h$, Representative images of dendritic spines from DIV21 hippocampal neurons expressing GFPtagged LASP2 truncation mutants ( $\Delta$ LIM, $\Delta$ Nebulin, $\Delta$ Linker, and $\Delta S H 3$ ) and mCherry. Scale bar, $5 \mu \mathrm{m}$. $\boldsymbol{i}-\boldsymbol{k}$, Effects of GFP-LASP2 mutants expression on dendritic spine density and morphology. Graphs show spine density ( $\boldsymbol{i}$; Kruskal-Wallis test: $p=0.0089, \mathrm{H}=13.55, \mathrm{df}=4$; Dunn's test: WT vs $\Delta$ LIM, $p=0.0227$; WT vs $\Delta$ Nebulin, $p=0.0338$; WT vs $\Delta$ Linker, $p>$ 0.9999; WT vs $\Delta S H 3, p=0.0880$ ), spine width (j; Kruskal-Wallis test: $p=0.0118, \mathrm{H}=12.89$, $\mathrm{df}=4$; Dunn's test: WT vs $\Delta \mathrm{LIM}, p=0.6478 ;$ WT vs $\Delta$ Nebulin, $p>0.9999 ;$ WT vs $\Delta$ Linker, $p>0.9999 ;$ WT vs $\Delta S H 3, p=0.4376)$, and percentage of spines with filopodial-like morphology ( $\boldsymbol{k}$; Kruskal-Wallis test: $p=0.2191, \mathrm{H}=5.744, \mathrm{df}=4$; Dunn's test: WT vs $\Delta$ LIM, $p>$ 0.9999 ; WT vs $\Delta$ Nebulin, $p>0.9999$; WT vs $\Delta$ Linker, $p=0.1266$; WT vs $\Delta$ SH3, $p>0.9999$; $n=1179$ spines from 17 cells for WT, 1182 spines from 17 cells for $\Delta$ LIM, 1144 spines from 18 cells for $\Delta$ Nebulin, 1429 spines from 21 cells for $\Delta$ Linker, and 1394 spines from 21 cells for $\Delta S H 3)$. All graphs represent data collected from three independent experiments. Error bars represent the SEM. ${ }^{*} p \leq 0.05,{ }^{* *} p \leq 0.01,{ }^{* * *} p \leq 0.001$ by one-way ANOVA with Tukey's multiple-comparisons test or Kruskal-Wallis test with Dunn's post hoc test.
}

Next, we investigated the role for these domains in dendritic arbor stabilization (Fig. 10a). Neurons expressing GFP-LASP2$\Delta \mathrm{SH} 3$ and GFP-LASP2- $\Delta$ Linker had dendritic arbors that were essentially indistinguishable from GFP-LASP2-WT neurons, with no detectable differences in dendritic complexity, branch number, or total dendritic length (Fig. 10b-e). This suggests that neither of these domains is required to promote dendrite complexity. Conversely, neurons expressing the LASP2- $\Delta$ LIM and LASP2- $\Delta$ Nebulin deletion mutants displayed a significant decrease in branch tip number, and an increase in mean branch length compared with LASP2-WT neurons (Fig. 10b-e). This indicates that the LIM domain and nebulin repeats are required to promote dendritic complexity. As expected, we observed no differences in the dendritic arbors of neurons expressing any of the LASP1 deletion mutants (Fig. 10f-j).

\section{Discussion}

Previous studies have indicated that both LASP1 and LASP2 are highly expressed in the fetal and adult CNS (Phillips et al., 2004; Terasaki et al., 2004; Zieseniss et al., 2008). LASP1 was shown to localize to the leading edge of growth cones during early development and then transition into dendritic spines where it associates with postsynaptic membranes during later development (Phillips et al., 2004). Single nucleotide polymorphisms and altered expression levels of LASP1 have also been associated with a number of neurological disorders such schizophrenia, autism, and bipolar disorder (Stone et al., 2007; Joo et al., 2013; Giusti et al., 2014). Finally, the upregulation of LASP1, but not LASP2, has previously been detected in response to nerve growth factor stimulation in rat PC-12 cells, a commonly used model for neurite outgrowth (Chen et al., 2008). However, to date, no studies have directly demonstrated a functional role for either gene in neurons. In this study, we present evidence that LASP1 and LASP2 differentially regulate postsynaptic dendrite and spine development. Knocking down LASP1 leads to defects in dendritic spine development, but does not affect dendritic arbor formation. LASP2 on the other hand, promotes dendritic spine stability, as well as the growth and complexity of dendritic arbors. Knockdown of LASP2 causes dramatic reductions in synaptic number and decreases the stability of both dendritic spines and dendritic branches. Using LASP2 truncation mutants, we found that the actin-binding LIM and nebulin repeats are essential for LASP2 function in neurons. Our findings provide the first evidence of a function for either LASP1 or LASP2 in neuronal and synaptic development.

LASP1 and LASP2 are members of the nebulin family of actinbinding proteins, which includes nebulin, N-RAP (nebulinrelated anchoring protein), and nebulette (Pappas et al., 2011). The nebulin family members are characterized by the presence of varying numbers of actin-binding nebulin repeats, from as few as two repeats in LASP1, up to 185 repeats for nebulin (Chu et al., 2016). Family members are capable of binding to a wide variety of F-actin structures, including lamellipodial actin bundles, stress fibers, sarcomeric thin filaments, $z$-discs, and intercalated discs. LASP1 and LASP2 differ from other nebulin family members due to the presence of both a LIM domain and an SH3 domain. SH3 domains are shared by a diverse array of structural and signaling proteins and typically mediate interactions with the proline-rich motifs of target proteins (Kurochkina and Guha, 2013). In nonneuronal cells, the SH3 domain and linker region of LASP1 and LASP2 are required for their recruitment to focal adhesions (Li et al., 2004; Panaviene and Moncman, 2007). The association of LASP1 to actin stress fibers was also shown to be indirect, via an 

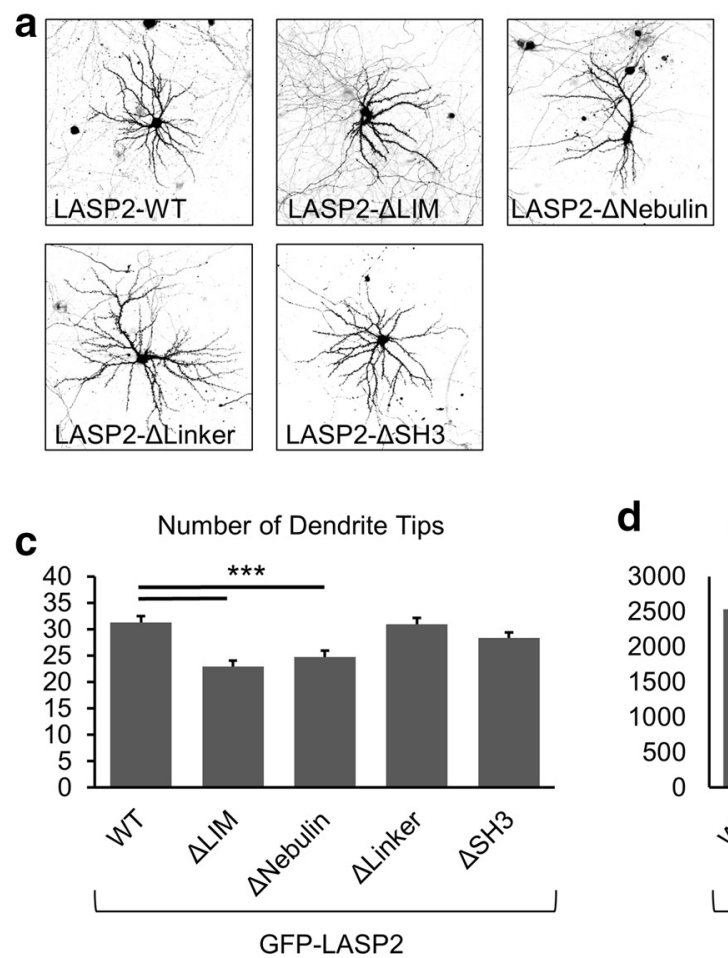

d

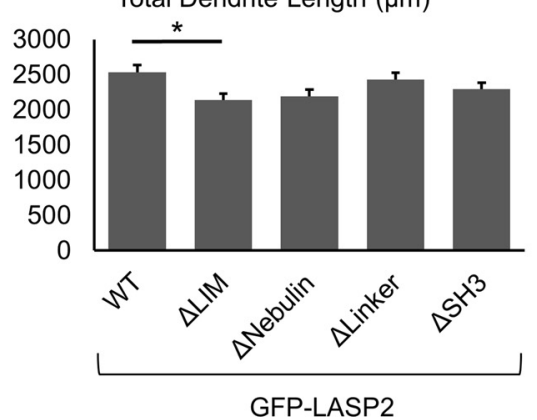

Sholl Analysis (LASP2 Deletion Mutants)

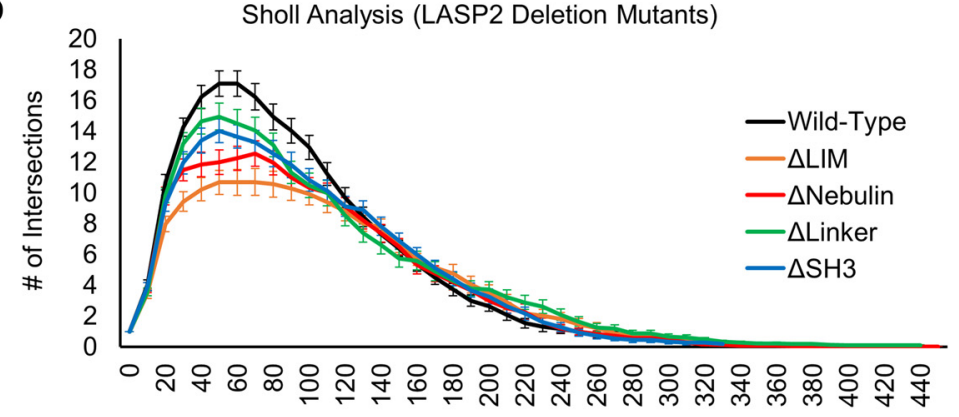

Distance from Soma $(\mu \mathrm{m})$

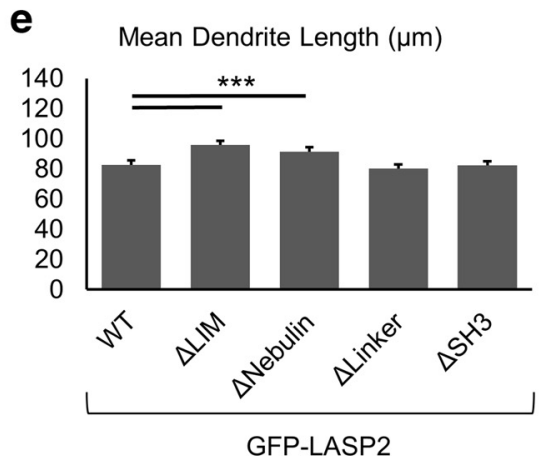

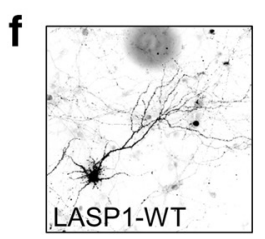
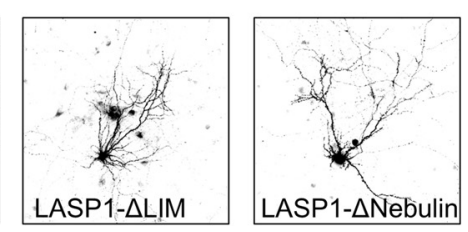

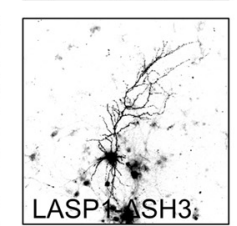

g

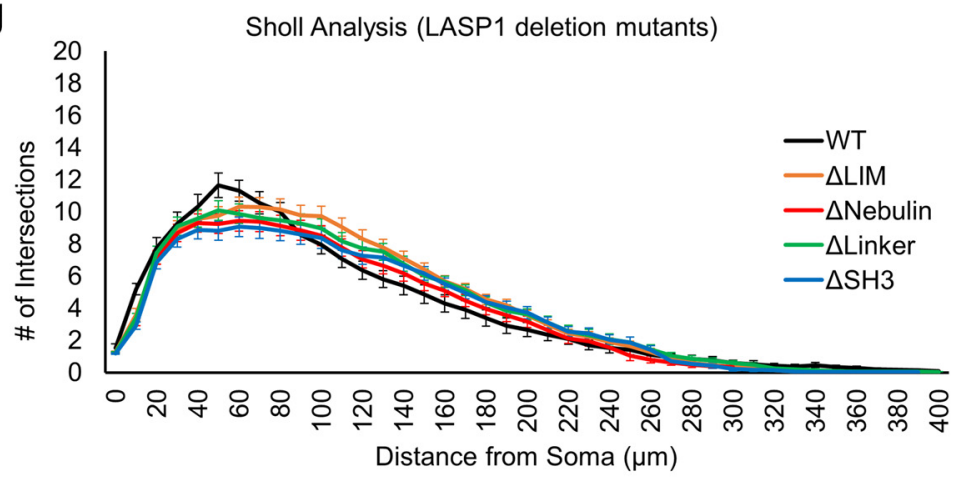

h

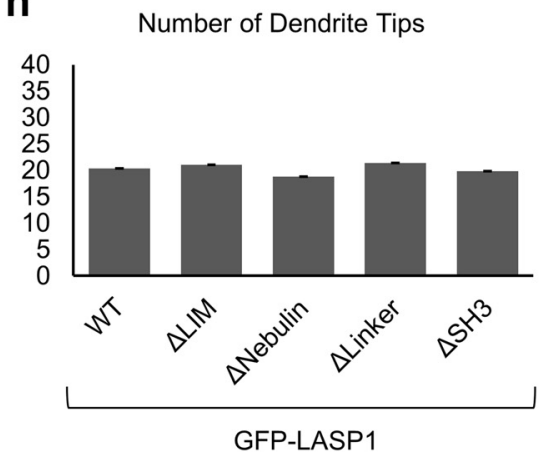

i

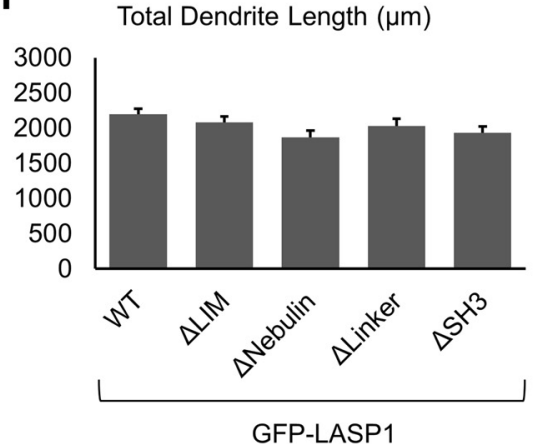

j

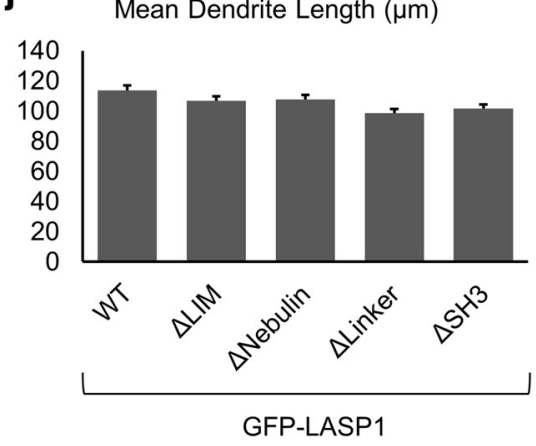

Figure 10. The LIM domain and nebulin repeats of LASP2 are required for dendritic arbor stabilization. $\boldsymbol{a}$, Images of DIV21 hippocampal neurons expressing GFP-tagged LASP2 deletion mutants for $10 \mathrm{~d}$. $\boldsymbol{b}$, Sholl analysis for GFP-LASP2 truncation mutants. c $-e$, Quantification of dendritic tip number ( $c$; Kruskal-Wallis test: $p<0.0001, \mathrm{H}=33.95$, df $=4$; Dunn's test:WT vs $\Delta$ LIM, $p<0.0001$; WT vs $\Delta$ Nebulin, $p=0.0015$; WT vs $\Delta$ Linker, $p>0.9999 ;$ WT vs $\Delta$ SH3, $p=0.6302$ ), total dendriticlength ( $d$; Kruskal-Wallis test: $p=0.0414, \mathrm{H}=9.941$, df $=4$; Dunn'stest:WTvs $\Delta$ LIM, $p=0.0245 ;$ WT vs $\Delta$ Nebulin, $p=$ 0.0889; WT vs $\Delta$ Linker, $p>0.9999 ;$ WT vs $\Delta$ SH3, $p=0.2172$ ), and mean dendritic branch length ( $\boldsymbol{e}$; Kruskal-Wallis test: $p<0.0001, \mathrm{H}=24.55$, df $=4$; Dunn's test: WT vs $\Delta$ LIM, $p=0.0017$; WT vs $\Delta$ Nebulin, $p=0.0478$; WT vs $\Delta$ Linker, $p>0.9999 ;$ WT vs $\Delta S H 3, p>0.9999 ; n=44$ cells for WT, 44 for $\Delta$ LIM, 45 for $\Delta$ Nebulin, 43 for $\Delta$ Linker, and 43 for $\Delta S H 3)$. f, Representative images of neurons expressing GFP-tagged LASP1 truncation mutants ( $\Delta$ LIM, $\Delta$ Nebulin, $\Delta$ Linker, and $\Delta S H 3$ ). $\boldsymbol{g}$, Sholl analysis showing no change in complexity for GFP-LASP1 truncation mutants. $\boldsymbol{h}$-j, Quantification of dendritic tip number ( $h$; Kruskal-Wallis test: $p=0.5087, \mathrm{H}=3.302, \mathrm{df}=4$; Dunn's test:WT vs $\Delta$ LIM, $p>0.9999 ;$ WT vs $\Delta$ Nebulin, $p>0.9999 ;$ WT vs $\Delta$ Linker, $p>0.9999 ;$ WT vs $\Delta S H 3, p>0.9999$ ), total dendritic length ( $i$; Kruskal-Wallis test: $p=0.4249, \mathrm{H}=3.863$, df $=4$; Dunn's test: WT vs $\Delta \mathrm{LIM}, p>0.9999 ;$ WT vs $\Delta$ Nebulin, $p=0.8399 ;$ WT vs $\Delta$ Linker, $p>0.9999 ;$ WT vs $\Delta S H 3, p>0.9999)$, and mean dendritic branch length (; Kruskal-Wallis test: $p=0.4576, \mathrm{H}=3.635$, df $=4$; Dunn's test:WT vs $\Delta$ LIM, $p>0.9999 ;$ WT vs $\Delta$ Nebulin, $p>0.9999 ;$ WT vs $\Delta$ Linker, $p=4887 ;$ WT vs $\Delta S H 3, p>0.9999 ; n=$ 41 cells for WT, 41 for $\Delta$ LIM, 40 for $\Delta$ Nebulin, 41 for $\Delta$ Linker, and 41 for $\Delta S H 3$ ). All graphs represent data collected from three independent experiments. Scale bars, $50 \mu \mathrm{m}$. $g$, Error bars for Sholl analysis represent $95 \% \mathrm{Cl}$; otherwise, error bars represent the SEM. ${ }^{*} p \leq 0.05,{ }^{* * *} p \leq 0.001$ by Kruskal-Wallis test with Dunn's post hoc test. 
SH3-dependent interaction with paladin (Rachlin and Otey, 2006). Here, we show that deletion of the SH3 domain causes a small but incomplete loss of LASP localization to spines, suggesting that it mediates some interactions with other proteins in spines. However, we found that both the LIM domain and the nebulin repeats are essential for LASP localization to spines, and are required for the role of LASP2 in dendritic spine and arbor development. Because it was previously shown that the LASP2 nebulin repeats alone are insufficient to bind F-actin without the LIM domain, it is not surprising that both domains are therefore required (Nakagawa et al., 2009).

The expression of the non-LASP nebulin family members is largely restricted to striated muscle, where they function as stabilizers of F-actin structures as well as scaffolds for various cytoskeletal assemblies (Pappas et al., 2011). The ability to stabilize F-actin structures has been demonstrated in several settings, including a study from the Gregorio laboratory that used an artificially truncated "mini-nebulin" to demonstrate that nebulin protects actin thin filaments from depolymerization by latrunculin A (Pappas et al., 2010). They also showed that the knockdown of nebulin increased the dynamics of actin thin filaments in cardiomyocytes (Pappas et al., 2010). A similar increase in actin dynamics could explain how the knockdown of LASP2 leads to increased spine and dendritic branch instability. LASP2 has previously been shown to bundle F-actin (Zieseniss et al., 2008), and this function provides a potential mechanism whereby LASP2 may be directly stabilizing F-actin in spines and dendrites. However, this does not explain why there are such dramatic differences between LASP1 and LASP2 function in neurons. Human LASP1 and LASP2 share $78.3 \%$ sequence similarity overall, and are especially conserved within the LIM domain (100\% similar) and the first two nebulin repeats $(98.5 \%$ similar). However, LASP2 contains a third nebulin repeat that is not found in LASP1. We hypothesize that the presence of the third nebulin repeat is what confers this LASP2-specific bundling activity. This is supported by earlier studies demonstrating the ability of six-repeat recombinant human nebulin fragments to bundle actin, and the inability of two repeats to bundle actin. Furthermore, previous studies have directly demonstrated that LASP2 bundles F-actin, while LASP1 cannot (Chew et al., 2002a; Zieseniss et al., 2008).

Alternatively, it is possible that LASP1 and LASP2 interact with F-actin in different ways, despite their high sequence similarity. For example, nebulin is capable of interacting with the pointed end-capping protein Tropomodulin 1 via its first three N-terminal nebulin repeats (McElhinny et al., 2001). In addition, the SH3 domain of nebulin has been shown to interact with the barbed end capping protein CapZ (Pappas et al., 2008). In this way, nebulin is able to cap actin filaments at both the pointed and barbed ends. This raises the possibility that either LASP1 or LASP2 may interact with a unique actin-capping or actinbinding protein that specifically orients it in a certain place or structure in spines. For example, LASP1 or LASP2 (but not the other) could be directed to the plus or minus end of actin filaments in spines, thereby conferring unique functions for each protein. It should be noted that within individual spines, there are multiple subspine actin domains with different properties and ultrastructure (Bosch et al., 2014; Chazeau et al., 2014).

In summary, LASP2 appears to function in the stabilization of protrusive structures such as dendritic spines and dendritic branches, likely via stabilization of the underlying actin cytoskeleton. LASP1 on the other hand appears to have a much smaller role in dendritic development under basal conditions. Considering that LASP1 (but not LASP2) was originally identified as a phosphoprotein, it is possible that LASP1 instead plays a role in synaptic plasticity/activity-dependent signaling. LASP1 is phosphorylated by cAMP- and cGMP-dependent protein kinases [protein kinase A (PKA) and PKG] as well as Abelson tyrosine kinase (Abl), and dephosphorylated by protein phosphatase $2 \mathrm{~B}$ (PP2B; Butt et al., 2003; Lin et al., 2004; Mihlan et al., 2013). PKA and PP2B in particular are both known as critical regulators of synaptic plasticity, and they may form a common signaling pathway with LASP1 at the synapse (Mansuy, 2003; Kandel, 2012). Furthermore, it has previously been shown that the phosphorylation of LASP1 affects its own subcellular localization to focal adhesions and affects the rate of cell migration in non-neuronal cells (Butt et al., 2003; Lin et al., 2004). This would raise the possibility that LASP2 plays a structural role in actin filament stabilization, whereas LASP1 could facilitate synaptic signaling and actin remodeling during synaptic plasticity.

\section{References}

Berry KP, Nedivi E (2017) Spine dynamics: are they all the same? Neuron 96:43-55.

Bliss KT, Chu M, Jones-Weinert CM, Gregorio CC (2013) Investigating lasp-2 in cell adhesion: new binding partners and roles in motility. Mol Biol Cell 24:995-1006.

Bosch M, Hayashi Y (2012) Structural plasticity of dendritic spines. Curr Opin Neurobiol 22:383-388.

Bosch M, Castro J, Saneyoshi T, Matsuno H, Sur M, Hayashi Y (2014) Structural and molecular remodeling of dendritic spine substructures during long-term potentiation. Neuron 82:444-459.

Bourne JN, Harris KM (2008) Balancing structure and function at hippocampal dendritic spines. Annu Rev Neurosci 31:47-67.

Butt E, Raman D (2018) New frontiers for the cytoskeletal protein LASP1. Front Oncol 8:391.

Butt E, Gambaryan S, Göttfert N, Galler A, Marcus K, Meyer HE (2003) Actin binding of human LIM and SH3 protein is regulated by cGMP- and cAMP-dependent protein kinase phosphorylation on serine 146. J Biol Chem 278:15601-15607.

Chazeau A, Mehidi A, Nair D, Gautier JJ, Leduc C, Chamma I, Kage F, Kechkar A, Thoumine O, Rottner K, Choquet D, Gautreau A, Sibarita JB, Giannone $G$ (2014) Nanoscale segregation of actin nucleation and elongation factors determines dendritic spine protrusion. EMBO J 33:27452764.

Cheadle L, Biederer T (2014) Activity-dependent regulation of dendritic complexity by semaphorin 3A through Farp1. J Neurosci 34:7999-8009.

Chen L, Maures TJ, Jin H, Huo JS, Rabbani SA, Schwartz J, Carter-Su C (2008) SH2B1beta (SH2-Bbeta) enhances expression of a subset of nerve growth factor-regulated genes important for neuronal differentiation including genes encoding urokinase plasminogen activator receptor and matrix metalloproteinase 3/10. Mol Endocrinol 22:454-476.

Chen Y, Sabatini BL (2012) Signaling in dendritic spines and spine microdomains. Curr Opin Neurobiol 22:389-396.

Chew CS, Chen X, Qin HY, Stoming T (2002a) New insights into second messenger regulation of parietal cell function by novel downstream signaling proteins. In: Mechanisms and consequences of proton transport (Urushidani T, Forte JG, Sachs G, eds), pp 185-195. Boston: Springer.

Chew CS, Chen X, Parente JA Jr, Tarrer S, Okamoto C, Qin HY (2002b) Lasp- 1 binds to non-muscle F-actin in vitro and is localized within multiple sites of dynamic actin assembly in vivo. J Cell Sci 115:4787-4799.

Chu M, Gregorio CC, Pappas CT (2016) Nebulin, a multi-functional giant. J Exp Biol 219:146-152.

Cline H, Haas K (2008) The regulation of dendritic arbor development and plasticity by glutamatergic synaptic input: a review of the synaptotrophic hypothesis. J Physiol 586:1509-1517.

Giusti L, Mantua V, Da Valle Y, Ciregia F, Ventroni T, Orsolini G, Donadio E, Giannaccini G, Mauri M, Cassano GB, Lucacchini A (2014) Search for peripheral biomarkers in patients affected by acutely psychotic bipolar disorder: a proteomic approach. Mol Biosyst 10:1246-1254.

Grabrucker A, Vaida B, Bockmann J, Boeckers TM (2009) Synaptogenesis of hippocampal neurons in primary cell culture. Cell Tissue Res 338:333341. 
Grunewald TG, Butt E (2008) The LIM and SH3 domain protein family: structural proteins or signal transducers or both? Mol Cancer 7:31.

Hotulainen P, Hoogenraad CC (2010) Actin in dendritic spines: connecting dynamics to function. J Cell Biol 189:619-629.

Joo J, Lee S, Nah SS, Kim YO, Kim DS, Shim SH, Hwangbo Y, Kim HK, Kwon JT, Kim JW, Song HY, Kim HJ (2013) Lasp1 is down-regulated in NMDA receptor antagonist-treated mice and implicated in human schizophrenia susceptibility. J Psychiatr Res 47:105-112.

Kadrmas JL, Beckerle MC (2004) The LIM domain: from the cytoskeleton to the nucleus. Nat Rev Mol Cell Biol 5:920-931.

Kandel ER (2012) The molecular biology of memory: cAMP, PKA, CRE, CREB-1, CREB-2, and CPEB. Mol Brain 5:14.

Koleske AJ (2013) Molecular mechanisms of dendrite stability. Nat Rev Neurosci 14:536-550.

Kurochkina N, Guha U (2013) SH3 domains: modules of protein-protein interactions. Biophys Rev 5:29-39.

Lai KO, Ip NY (2013) Structural plasticity of dendritic spines: the underlying mechanisms and its dysregulation in brain disorders. Biochim Biophys Acta 1832:2257-2263.

Lei W, Omotade OF, Myers KR, Zheng JQ (2016) Actin cytoskeleton in dendritic spine development and plasticity. Curr Opin Neurobiol 39: $86-92$.

Li B, Zhuang L, Trueb B (2004) Zyxin interacts with the SH3 domains of the cytoskeletal proteins LIM-nebulette and lasp-1. J Biol Chem 279:2040120410.

Lin YC, Koleske AJ (2010) Mechanisms of synapse and dendrite maintenance and their disruption in psychiatric and neurodegenerative disorders. Annu Rev Neurosci 33:349-378.

Lin YH, Park ZY, Lin D, Brahmbhatt AA, Rio MC, Yates JR 3rd, Klemke RL (2004) Regulation of cell migration and survival by focal adhesion targeting of lasp-1. J Cell Biol 165:421-432.

Longair MH, Baker DA, Armstrong JD (2011) Simple neurite tracer: open source software for reconstruction, visualization and analysis of neuronal processes. Bioinformatics (Oxford) 27:2453-2454.

Mansuy IM (2003) Calcineurin in memory and bidirectional plasticity. Biochem Biophys Res Commun 311:1195-1208.

McElhinny AS, Kolmerer B, Fowler VM, Labeit S, Gregorio CC (2001) The $\mathrm{N}$-terminal end of nebulin interacts with tropomodulin at the pointed ends of the thin filaments. J Biol Chem 276:583-592.

Mihlan S, Reiß C, Thalheimer P, Herterich S, Gaetzner S, Kremerskothen J, Pavenstädt HJ, Lewandrowski U, Sickmann A, Butt E (2013) Nuclear import of LASP-1 is regulated by phosphorylation and dynamic proteinprotein interactions. Oncogene 32:2107-2113.

Nakagawa H, Suzuki H, Machida S, Suzuki J, Ohashi K, Jin M, Miyamoto S, Terasaki AG (2009) Contribution of the LIM domain and nebulinrepeats to the interaction of lasp-2 with actin filaments and focal adhesions. PLoS One 4:e7530.

Nimchinsky EA, Sabatini BL, Svoboda K (2002) Structure and function of dendritic spines. Annu Rev Physiol 64:313-353.

Orth MF, Cazes A, Butt E, Grunewald TG (2015) An update on the LIM and SH3 domain protein 1 (LASP1): a versatile structural, signaling, and biomarker protein. Oncotarget 6:26-42.
Panaviene Z, Moncman CL (2007) Linker region of nebulin family members plays an important role in targeting these molecules to cellular structures. Cell Tissue Res 327:353-369.

Pappas CT, Bhattacharya N, Cooper JA, Gregorio CC (2008) Nebulin interacts with CapZ and regulates thin filament architecture within the Z-disc. Mol Biol Cell 19:1837-1847.

Pappas CT, Krieg PA, Gregorio CC (2010) Nebulin regulates actin filament lengths by a stabilization mechanism. J Cell Biol 189:859-870.

Pappas CT, Bliss KT, Zieseniss A, Gregorio CC (2011) The nebulin family: an actin support group. Trends Cell Biol 21:29-37.

Parrish JZ, Emoto K, Kim MD, Jan YN (2007) Mechanisms that regulate establishment, maintenance, and remodeling of dendritic fields. Annu Rev Neurosci 30:399-423.

Peng YR, He S, Marie H, Zeng SY, Ma J, Tan ZJ, Lee SY, Malenka RC, Yu X (2009) Coordinated changes in dendritic arborization and synaptic strength during neural circuit development. Neuron 61:71-84.

Phillips GR, Anderson TR, Florens L, Gudas C, Magda G, Yates JR 3rd, Colman DR (2004) Actin-binding proteins in a postsynaptic preparation: lasp-1 is a component of central nervous system synapses and dendritic spines. J Neurosci Res 78:38-48.

Rachlin AS, Otey CA (2006) Identification of palladin isoforms and characterization of an isoform-specific interaction between lasp- 1 and palladin. J Cell Sci 119:995-1004.

Rochefort NL, Konnerth A (2012) Dendritic spines: from structure to in vivo function. EMBO Rep 13:699-708.

Scott EK, Luo L (2001) How do dendrites take their shape? Nat Neurosci $4: 359-365$.

Segal M (2005) Dendritic spines and long-term plasticity. Nat Rev Neurosci 6:277-284.

Sin WC, Haas K, Ruthazer ES, Cline HT (2002) Dendrite growth increased by visual activity requires NMDA receptor and rho GTPases. Nature 419:475-480.

Stölting M, Wiesner C, van Vliet V, Butt E, Pavenstädt H, Linder S, Kremerskothen J (2012) Lasp-1 regulates podosome function. PLoS One 7:e35340.

Stone JL, Merriman B, Cantor RM, Geschwind DH, Nelson SF (2007) High density SNP association study of a major autism linkage region on chromosome 17. Hum Mol Genet 16:704-715.

Terasaki AG, Suzuki H, Nishioka T, Matsuzawa E, Katsuki M, Nakagawa H, Miyamoto S, Ohashi K (2004) A novel LIM and SH3 protein (lasp-2) highly expressing in chicken brain. Biochem Biophys Res Commun 313:48-54.

Van Aelst L, Cline HT (2004) Rho GTPases and activity-dependent dendrite development. Curr Opin Neurobiol 14:297-304.

Yoshihara Y, De Roo M, Muller D (2009) Dendritic spine formation and stabilization. Curr Opin Neurobiol 19:146-153.

Zieseniss A, Terasaki AG, Gregorio CC (2008) Lasp-2 expression, localization, and ligand interactions: a new Z-disc scaffolding protein. Cell Motil Cytoskeleton 65:59-72.

Ziv NE, Smith SJ (1996) Evidence for a role of dendritic filopodia in synaptogenesis and spine formation. Neuron 17:91-102. 